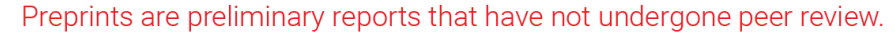 \\ They should not be considered conclusive, used to inform clinical practice, or referenced by the media as validated information. \\ USP39 Promotes Malignant Proliferation and Angiogenesis of Renal Cell Carcinoma by Inhibiting VEGF-A Alternative Splicing
}

\section{Xiu-wu Pan}

Second Military Medical University

\section{Da Xu}

Second Military Medical University

\section{Wenjin Chen}

Second Military Medical University

Jia-xin Chen

Second Military Medical University

Jian-qing Ye

Second Military Medical University

\section{Si-shun Gan}

Second Military Medical University

\section{Wang Zhou}

Second Military Medical University

\section{Song Xu}

Shanghai Seventh People's Hospital

\section{Lei Shi}

Qingdao University Medical College

Xingang Cui ( $\square$ cuixingang@smmu.edu.cn )

Second Military Medical University https://orcid.org/0000-0002-0112-5580

\section{Research}

Keywords: renal cell carcinoma, USP39, VEGF-A alternative splicing, SRPK1, SRSF1

Posted Date: September 17th, 2020

DOI: https://doi.org/10.21203/rs.3.rs-75498/v1

License: (1) (1) This work is licensed under a Creative Commons Attribution 4.0 International License.

Read Full License 


\section{Abstract}

Objective: To investigate the function and mechanism of USP39 in promoting malignant proliferation and angiogenesis of RCC.

Methods: We applied ONCOMINE database to analyze the correlation between USP39 expression level and the clinical characteristics of RCC. The effect of USP39 on RCC was evaluated by MTT assay, cell cycle analysis, colony formation assay and tubule formation assay. The interaction between USP39 and VEGF-A alternative splicing was assessed by co-immunoprecipitation and Western blot assays.

Results: The mRNA expression level of USP39 in RCC was significantly higher than that in normal renal tissue $(P<0.001)$, and negatively correlated with the survival rate of RCC patients $(P<0.01)$. Silencing of USP39 in 786-O and ACHN cells obviously inhibited cell proliferation and colony formation, and induced $S$ phase arrest. USP39 overexpression significantly increased the number of tubules $(P<0.05)$ and branches $(P<0.01)$ formed by HUVEC cells, and USP39 knockdown produced an opposite effect $(P<0.05)$. The USP39 (101-565) fragment directly mediated its binding to SRSF1 and SRPK1, and promoted the phosphorylation of SRSF1 to regulate VEGF-A alternative splicing. USP39 knockdown upregulated the expression of VEGF-A165b, and USP39 overexpression downregulated the expression of VEGF-A165b significantly (both $\mathrm{P}<0.05)$.

Conclusion: USP39 acted as a pro-tumor factor by motivating the malignant biological processes of RCC, probably through promoting VEGF-A alternative splicing and regulating SRSF1 and SRPK1. USP39 may prove to be a potential therapeutic target for RCC.

\section{Graphical Abstract}

USP39 directly interacts with SRPK1 to promote SRSF1 phosphorylation and downregulate the generation of VEGF-A165b variable splicing, leading to angiogenesis and serving as a pro-tumor factor of RCC.

\section{Research In Context}

\section{Evidence before this study}

Previous studies have shown that the benefits of targeted therapy for renal cell carcinoma (RCC) are dramatically impaired due to drug resistance. Rapid disease progression and poor prognosis occur in patients with drug resistance. USP39 upregulation was correlated with the development of several other tumors. As yet, the mechanism to explain whether USP39 plays a role in renal cell carcinoma has not been identified.

\section{Added value of this study}


We applied ONCOMINE database to analyze the correlation between USP39 expression level and the clinical characteristics of RCC. The interaction between USP39 and VEGF-A alternative splicing was assessed by Co-immunoprecipitation (Co-IP) and Western blot assays. The mRNA expression level of USP39 in RCC is significantly higher than that of normal renal tissue, and negatively correlated with the survival rate of RCC patients. Silencing of USP39 in 786-O and ACHN cells obviously inhibited cell proliferation and colony formation with inducing $S$ phase arrest. The number of tubules and branches formed by HUVEC cells increased significantly by USP39 overexpressing, while decreased markedly by USP39 knocking down. The USP39 (101-565) fragment mediates directly binding to SRSF1 and SRPK1, and promotes the phosphorylation of SRSF1 to regulate VEGF-A alternative splicing. The expression of VEGF-A165b significantly was upregulated by knockdown of USP39, while significantly downregulated by overexpression of USP39. Overall, USP39 serves as a pro-tumor factor motivating malignant biological processes of RCC, probably by promoting VEGF-A alternative splicing through regulating SRSF1 and SRPK1.

\section{Implications of all the available evidence}

We firstly confirmed the predictive value of USP39 on survival, recurrence, metastasis in RCC patients, especially for those with low TNM stage, and TNM stage combined with USP39 expression is prior to the single factor. We have discovered a new regulatory network among USP39, SRPK1, SRSF1 and VEGFA165b, which can promote the tumorigenesis and development of RCC. The downregulation of USP39 could inhibit RCC cells proliferation and progression, suggesting that USP39 potentially become the inhibition point for RCC. Our study provides a theoretical basis for new targets to the treatment of RCC.

\section{Introduction}

Renal cell carcinoma (RCC) is one of the most lethal malignancies of the genitourinary system causing approximately 140000 deaths each year worldwide [1, 2]. About 70\% RCC patients were diagnosed with localized RCC in the early stage, which could be potentially cured by radical nephrectomy [3]. However, the other RCC patients may have developed metastasis at the initial diagnosis [4]. In addition, 20-40\% RCC patients may experience recurrence or metastasis one or two years after the initial surgery with poor survival and prognosis [5]. Conventional therapies including radiotherapy or chemotherapy have limited and disappointing efficacy for advanced RCC patients [6]. Although the advent of targeted therapy including tyrosine kinase inhibitors (TKIs) has improved overall survival (OS) and progression-free survival (PFS) of patients with advanced RCC, drug resistance and rapid disease progression occurred frequently [7]. Therefore, it is an urgent task to gain novel insights into the mechanisms and therapeutic targets of RCC.

Accumulating studies have demonstrated that mutations or activities of RNA splicing-related factors participate in the development and progression of various malignant tumors [8,9]. Ubiquitin-specific peptidase 39 (USP39), first found in yeast, is known as a type of protein associated with the assembly process of spliceosomal snRNP during pre-mRNA maturation [10,11]. The molecular structure of USP39 
consists of three domains: RS-like domain of $\mathrm{N}$-terminal, ubiquitin binding domain of zinc finger protein (ZnF-UBP) in the middle, and ubiquitin-specific protease (USP) domain of C-terminal, without the activity of ubiquitin enzyme $[12,13]$. It was reported that USP39 upregulation was correlated with the development of medullary thyroid carcinoma (MTC) and human hepatocellular carcinoma (HCC) $[14,15]$. It was found in our previous study that USP39 knockdown could inhibit the abnormal proliferation of prostate cancer cells by inhibiting the splicing maturation and transcriptional prolongation of EGFR mRNA [16]. Other studies have also reported that USP39 serves as the pro-tumor factor in many malignant tumors such as gastric cancer [17], osteosarcoma [18], lung cancer [19], glioma [20], and breast cancer [21]. In addition, USP39 knockdown was found to inhibit RCC progression through blocking Akt/ERK pathways [22]. However, the role of USP39 on splicing complex regulation in RCC progression remains unclear.

Tumor neovascularization originates from the abnormal pathological process of angiogenesis due to the imbalance between promoters and inhibitors [23]. Overexpression of vascular endothelial growth factor (VEGF), especially VEGF-A, has been documented as a stimulator of tumor angiogenesis [24]. VEGF can produce different isoforms through mRNA splicing including VEGFA-165b [25], which is believed to be an anti-angiogenetic factor and downregulated in RCC, prostate cancer, colorectal cancer and melanoma [2629]. It has been reported that Serine/Arginine-Rich Protein Specific Kinase 1 (SRPK1) could phosphorylate Serine/Arginine-Rich Splicing Factor 1 (SRSF1) to promote VEGF-A splicing to generate VEGF-A165 (proangiogenesis) and VEGF-A165b (anti-angiogenesis ) [30-32]. It is therefore hypothesized that VEGF-165b or other anti-angiogenic splicing isoforms may become a promising therapeutic target or mediator of malignant tumors. However, the biological function and underlying mechanisms of VEGF splicing in RCC need to be elucidated.

The aim of the present study was to investigate the role of USP39 in RCC cell proliferation, malignant progression and angiogenesis and the potential mechanism of VEGF-A alternative splicing, in an attempt to gain deeper insights into the molecular mechanism underlying the development of RCC and provide new clues for exploring molecular targeted therapies of RCC.

\section{Materials And Methods}

\subsection{Ethics statement}

\subsection{Bioinformatic data analysis}

A series of survival data, expression level data, clinical characteristics were obtained from ONCOMINE database (www.oncomine.org) by using the following search terms: 'USP39', 'Cancer vs. Normal Analysis', 'Kidney Cancer' and 'mRNA'. Two datasets (Gumz Renal dataset and Jones Renal dataset) including RCC vs. normal kidney tissues were used to analyze the expression of USP39 in RCC and normal kidney tissues [33]. One dataset (Zhao Renal dataset) with 176 RCC tissues was used to explore the effect of USP39 on survival analysis [34]. Correlation analysis of USP39 and VEGF-A was performed on the Gumz Renal[31]. All data are reported Log2 Median-Centered intensity in the Oncomine database. 


\subsection{Cell culture and treatments}

A498, 769P, 786-O, ACHN, Caki-1, Caki-2, 293T and human umbilical vein endothelial (HUVEC) cells were purchased from the Cell Bank of Shanghai Academy of Life Sciences, the Chinese Academy of Sciences. Cells were cultured with 1640 or DMEM $+10 \%$ fetal bovine serum (FBS) $+1 \%$ penicillin double antibody at $37^{\circ} \mathrm{C}$ and $5 \% \mathrm{CO} 2$.

Recombinant plasmids pcDNA3-USP39 were constructed to overexpress USP39 in our laboratory. The two truncated forms of USP39, one with amino acids (AA) 1-100 (containing the RS-like domain, USP39 (1-100)) and the other with AA 101-565 (containing the ZnF, UCH1, and UCH2 domains, USP39 (101-565)), were acquired from the Key Laboratory of Cell Differentiation and Apoptosis of the National Ministry of Education (Shanghai Jiaotong University School of Medicine, Shanghai, China)[35]. Lentiviral USP39 knockdown plasmids (Lv-shUSP39, ShRNA sequences: 5'-GATTTGGAAGAGGCGAGATAA-3') were prepared by Hollybio Biotechnology Company (Shanghai, China). Empty plasmids (Con) Lentiviral vector with nonspecific shRNA (Lv-shCon) were used as controls. Lentiviruses were constructed in 293T cells according to the manufacturer's method. The knockdown and overexpression efficiency was assessed by RT-PCR and Western blot.

\subsection{MTT assay}

Cells with stable knockdown of USP39 were cultured in a 96-well plate at 2000 cells/well for Day 1, 2, 3, 4 and 5. At each time point, cells were incubated with 3-(4, 5-dimethylthiazol-2-yl)-2,5-diphenyltetrazolium bromide (MMT) solution for $4 \mathrm{~h}$ at $37^{\circ} \mathrm{C}$ and was terminated by acidic isopropanol solution. Half an hour later, cell viability was measured by $595 \mathrm{~nm}$ absorbance to acquire OD values. OD values of each day were used to draw the MTT growth curve. Each experiment was performed in triplicate.

\subsection{Plate colony formation assay}

Cells with stable knockdown of USP39 were cultured in a 6-well plate at 400 cells/well at $37^{\circ} \mathrm{C}$ for 14 days with the culture medium replaced at 3-day intervals. Cell colonies were fixed with $4 \%$ paraformaldehyde for $30 \mathrm{~min}$ and then stained under GIEMSA for $20 \mathrm{~min}$. Each cell colony was counted under a light microscope and photographed with a digital camera.

\subsection{Flow cytometric assay and cell cycle detection}

According to manufacturer's instructions, cells were centrifuged, resuspended with PBS, fixed by addition of proof ethanol to a final ratio at $66 \%$, incubated on ice for $15 \mathrm{~min}$, resuspended in a working solution with 500ul Propidium lodide (PI) buffer, 25ul PI (20x) and 5ul RNase A (50x), and incubated again at 37 ${ }^{\circ} \mathrm{C}$ for $40 \mathrm{~min}$. Flow cytometric analysis was performed using a flow cytometer (BD Biosciences, USA). Red fluorescence was detected at the excitation wavelength $488 \mathrm{~nm}$, and the laser emission was detected at the same time.

\subsection{Tubule formation assay}


After 24-h serum-free culture, cell supernatants of 786-0 and ACHN cells with knockdown or overexpressiom USP39 were collected. After addition of $50 \mu \mathrm{L}$ Matrigel solution to each well of the 96well plate, cells were incubated at $37^{\circ} \mathrm{C}$ for $1 \mathrm{~h}$. HUVECs were resuspended with the collected cell supernatant or serum-free 1640 medium at $1 \times 10^{5} \mathrm{cells} / \mathrm{mL}$ after serum-starvation overnight. The resuspended HUVECs $(100 \mu \mathrm{L})$ were added to the Matrigel-coated wells and incubated for $8 \mathrm{~h}$ at $37^{\circ} \mathrm{C}$. Subsequently, HUVECs were imaged with an inverted fluorescence microscope (CKX41, Olympus, Japan). ImageJ was applied to analyze the number of meshes, the number of branches, and the tube length.

\subsection{Quantitative real-time PCR (RT-qPCR)}

Total RNA was extracted with Trizol reagents (Invitrogen) and cDNA was obtained using First-Strand cDNA Synthesis Kit (Invitrogen). The resulting cDNA was subjected to RT-qPCR with the indicated primer sets. RT-qPCR analysis was conducted by Power SYBR Green PCR Master Mix (Applied Biosystems, Foster City, CA, USA). Relative gene expression was normalized to GAPDH with the $2^{-\triangle \triangle C T}$ assay. The primer sequences were used: for USP39, 5'-GCCAGCAGAAGAAAAAGAGC-3' (forward) and 5'GCCATTGAACTTAGCCAGGA-3' (reverse); for VEGF-A, 5'- GCACATAGGAGAGATGAGCTTCC-3' (forward) and 5'- CTCCGCTCTGAACAAGGCT-3' (reverse) for $\beta$-actin, 5'-ATCGTGCGTGACATTAAGGAG-3' (forward) and 5'-AGGAAGGAAGGCTGGAAGAG-3' (reverse). Values were normalized to those of Actin.

\subsection{Western blot}

Cells with stable overexpression or knockdown of USP39 were lysed using RIPA lysis buffer (Beyotime, China), and the protein concentration was measured by BCA assay (Beyotime). Samples were prepared in SDS sample loading buffer, and transferred to the PVDF membrane. The main antibodies of Western blot were anti-USP39 (Abcam), anti-SRSF1 (Abcam), anti-SRPK1 (Santa Cruz), anti-VEGF 165b (R\&D), and anti-VEGF-A (Abcam). The membrane was blocked by $5 \%$ milk (nonfat) in TBS $(0.05 \%$ Tween 20$)$ at room temperature for $1 \mathrm{~h}$, and incubated with the primary antibodies for $2 \mathrm{~h}$ and with HRP-conjugated IgG (rabbit). Western blotting detection system (Tanon) was applied to detect Chemiluminescence of the membrane.

\subsection{Co-immunoprecipitation (CO-IP) analysis}

Cells with stable overexpression or knockdown of USP39 were collected by RIPA buffer. Immunoprecipitation was conducted with anti-HA (Abcam) or anti-SRPK1 (Santa Cruz) or anti-Panphospho-SR (Santa Cruz). By incubation with protein A agarose (Santa Cruz), the antibodies were removed. Proteins were prepared and separated by 10\% SDS-PAGE. The interaction between USP39 and SRPK1/SRSF1 was analyzed by Western blot using anti-Flag tag (Abcam) tag or anti-SRSF1 (Abcam).

\subsection{Statistical analysis}

All data were analyzed by SPSS version 22.0 (IBM corporation) and processed by GraphPad Prism 8.0. Independent sample t-test was used for comparison between groups. $\mathrm{P}<0.05$ was considered 


\section{Role Of The Funding Source}

The sponsors of the study had not any role in study design, data collection, data analysis, interpretation, and writing of the report. The corresponding author had full access to all the data in the study and had final responsibility for the decision to submit for publication.

\section{Results}

\subsection{USP39 is highly expressed in clear cell renal cell carcinoma (ccRCC) and negatively correlated with survival of RCC patients.}

To confirm the correlation between the expression level of USP39 in RCC and the prognosis of RCC patients, we retrieved the expression level of USP39 in all types of RCC and normal renal tissues through ONCOMINE database. The expression of USP39 in RCC tissue was significantly higher than that in normal renal tissue $(P<0.001)(F i g .1 A)$. In addition, the expression level of USP39 was associated with the malignant degree of RCC, with the highest USP39 expression in CCRCC vs. normal renal tissue $(P<0.05)$ (Fig. 1B).

We further investigated the survival data in the zhao database of ONCOMINE. As showed in Table1, patients with high expression of USP39 had a significantly lower survival rate $(P<0.01)$ and a shorter median survival duration as compared with those in patients with low expression of USP39 $(P<0.01)$, indicating that the expression level of USP39 was negatively correlated with the survival rate of RCC patients. Patients with high expression of USP39 had poorer survival prognosis ( $P=0.006)$ (Fig $1 \mathrm{C})$, and the expression level of USP39 in patients who died at 5 years was significantly higher than that in patients who survived $(P<0.05)$ (Fig 1D). In addition, we conducted a comparative analysis based on the stages of renal cancer patients and the expression level of USP39, and found that high expression of USP39 predicted poor survival prognosis in patients with a low TNM stage, while a high TNM stage showed no statistical difference in USP39 expression ( $P=0.001, P=0.440)$ (Fig $1 E, F)$. Concerning the pathological grade, USP39 predicted poor survival prognosis in patients with low Fuhrman grade, while patients with high Fuhrman grade showed no statistical difference in USP39 expression (Fig 1G,H).

Meanwhile, we performed univariate and multivariate analyses to determine whether the expression of USP39 was an independent risk factor for survival of RCC patients. It was found that USP39 expression, TNM stage and the WHO performance status were independent risk factors for OS of RCC patients (Table 2, Fig 2A). Considering that the high expression of USP39 predicted poor prognosis in patients with low TNM stage and TNM stage is currently recognized as an indicator for predicting survival and prognosis of patients with renal cell carcinoma, we hypothesized whether TNM stage combined with USP39 expression could improve the ability to predict prognosis in RCC patients. We used ROC curve to analyze the predictive ability of single index TNM stage, USP39 expression and combined indexes. The results 
suggested that combined TNM stage and USP39 expression had a higher predictive ability than a single index $(\mathrm{P}<0.0001)$ (Fig 2B). For patients with low TNM stage, the predictive ability of USP39 expression was stronger than that of TNM stage, and the combination of the two indexes could significantly improve the ability of predicting survival of RCC patients $(\mathrm{P}<0.001)$ (Fig $2 \mathrm{C})$.

\subsection{Stable knockdown of USP39 in 786-0 and ACHN cell lines}

We chose 786-O and ACHN with relatively high expression of USP39 as candidate target cell lines by comparing the expression of USP39 in six RCC cell lines (Fig 3A,B). Then, Lentivirus-introduced shRNA (Lv-shUSP39) was used to silence USP39 expression in 786-0 and ACHN cells (Fig. 3C) . Western blotting and qRT-PCR were used to verify the efficiency of USP39 silencing. The results showed that the protein and mRNA expression levels of USP39 after knockdown were significantly lower than those of the control group $(P<0.001)$. Thus, $786-0$ and ACHN cell lines with USP39 knockdown were successfully prepared (Fig 3D-E).

\subsection{USP39 knockdown inhibits proliferation and colony formation by inducing $S$ arrest in 786-0 and ACHN cell lines}

To explore the change of proliferation after USP39 knockdown in 786-0 and ACHN cells, we applied MTT assay and colony formation assay. As shown in Fig 3, the MTT growth curve indicated that the cell proliferation was significantly inhibited by USP39 silencing compared with the control group (Fig. 3F,G). And the results of cell colony formation assay showed that the number of the clones formed after knockdown of USP39 was significantly lower than that in the control group, and had statistical significance $(\mathrm{P}<0.001)(\mathrm{Fig} .3 \mathrm{H}-\mathrm{J})$. The results of MTT assay and colony formation assay showed that knockdown of USP39 could significantly inhibit the malignant proliferation of RCC cells. Moreover, we performed FACS to analyze cell cycle distribution and evaluate the potential reason for the inhibition of proliferation. The results revealed that $S$ arrest occurred due to USP39 silencing $(P<0.05)($ Fig $4 A-C)$. These findings demonstrated that USP39 served as a strong pro-tumor factor in the malignant proliferation of RCC.

\subsection{Knockdown or overexpression of USP39 either inhibits or promotes tubule formation in vascular endothelial cells.}

RCC malignant proliferation is known to be correlated with tumor angiogenesis [36]. To investigate the effect of USP39 on the angiogenesis of vascular endothelial cells, we also overexpressed or knocked down USP39 in HUVEC, and then carried out tubule formation assay. It was found that USP39 overexpression significantly enhanced the tubule formation ability of HUVEC $(P<0.05)$, and increased the number of branches formed $(P<0.01)$ (Fig 4D-G). After knockdown of USP39, the tube formation ability of HUVEC was inhibited $(P<0.01$ ) (Fig 4D-G), and the number of branches was decreased correspondingly $(P<0.01)$ (Fig 4D-G). These findings indicated that knockdown or overexpression of USP39 could either inhibit or promote angiogenesis of endothelial cells. 


\subsection{The USP39 (101-565) fragment plays a biological role by binding and regulating SRSF1 and SRPK1.}

To explore the potential mechanism of USP39-mediated angiogenesis and malignant proliferation, affinity purification-mass spectrometry (AP-MS) was used to explore the interactions between USP39 and the potential target protein. It was found that USP39 combined with SRPK1 and SRSF1, which are regarded as key splicing factors involved in the alternative splicing of VEGF-A (Table S1). To verify protein-protein interaction networks, Co-IP was carried out. As described by AP-MS, USP39 interacted with SRPK1 and SRSF1, respectively (Fig 5A,B).

The molecular structure of USP39 consists of four domains: Arginine-serine-rich (RS)-like domain, ZnF domain, UCH1 domain, and UCH2 domain [10]. To determine which USP39 domain binds to SRPK1 and SRSF1, we received two truncated forms of USP39, including one RS-like domain (USP39 1-100) and one ZnF-UCH1-UCH2 complex domains (USP39 101-565) (Fig 5C). Co-IP assay of USP39 truncated domain was performed with SRPK1 and SRSF1. The binding site of SRPK1 was located in the ZnF-UCH1-UCH2 complex domains (USP39 101-565), not in the RS-like domain (Fig 5D). The same binding site was found in the interaction between USP39 and SRSF1 (Fig 5E). These results indicated that USP39 acted through binding the USP39 (101-565) domains to SRPK1/SRSF1.

It has been reported that SRPK1 functions by regulating the phosphorylation of SRSF1 to promote VEGFA alternative splicing [37]. To verify the regulatory effect of USP39 on SRPK1/SRSF1, we conducted Co-IP assay on 786-0 and ACHN cells after knockdown and overexpression of USP39. It was found that overexpression of USP39 could enhance the interaction between SRPK1 and SRSF1 (Fig. 6A). On the contrary, knockdown of USP39 could weaken the interaction between SRPK1 and SRSF1 (Fig. 6B). In addition, there was stronger phosphorylation of SRSF1 in 786-O and ACHN cells with overexpression of USP39, and knockdown of USP39 in RCC cells could inhibit phosphorylation of SRSF1 (Fig. 6C, D). These findings indicated that USP39 affected the phosphorylation of SRSF1 by regulating the interaction between SRPK1 and SRSF1.

\subsection{Knockdown or overexpression of USP39 either upregulates or downregulates VEGF-A165b expression.}

It was reported that SRPK1 directly acted on SRSF1 to promote the splicing of VEGF-A and enhance the production of VEGF-A165 and VEGF-A165b [32]. Cancer cells with knockdown of SRPK1 increased the expression of VEGF-A165b and reduced the expression of VEGF-A165 [30]. To verify the effect of USP39 on VEGF-A, the expression of VEGF-A and VEGF-A165b was analyzed by 786-0 cells with overexpression or knockdown of USP39. As shown in Fig 6E, RCC cells with knockdown of USP39 upregulated the expression of VEGF-A165b. On the contrary, RCC cells with overexpression of USP39 downregulated the expression of VEGF-A165b. It is worth noting that the splicing isomer of VEGF-A165b could inhibit endothelial cell angiogenesis [29], which also supports the result of the above-mentioned tubule formation assay.

\section{Discussion}


Kidney cancer ranks the top 10 common malignancies among all types of tumors, and RCC accounts for $85 \%$ of all kidney cancers [4] and $5 \%$ of epithelial malignant tumors diagnosed every year [38]. The median survival time of patients with metastatic RCC is only 12 months with a 5-year survival rate of less than $10 \%$ [39]. In addition, early diagnosis and prognostic judgement of RCC remain a challenge due to the lack of specific manifestations and a relatively high postoperative recurrence rate $[40,41]$. It is reported in the literature that USP39 acts as a type of pro-tumor gene. Wen et al [33] reported that overexpression of USP39 could promote the malignant proliferation of prostate cancer cells. Wang et al [76] also found that USP39 was highly expressed in breast cancer cells, and down-regulation of USP39 could significantly reduce the proliferation and colony formation of breast cancer cells. However, these studies failed to explain the role of USP39 in early stage RCC (TNM stage I-II or Fuhrman grade 1-2). We demonstrated that the expression of USP39 was positively correlated with the survival rate of RCC patients. High expression of USP39 could predict poor prognosis and played a significant role in patients with low TNM stage (TNM stage I-II) and low Fuhrman grade (Fuhrman grade 1-2). In addition, our univariate and multivariate analyses showed that the expression of USP39 was an independent risk factor for OS of RCC patients. The predictive value of TNM stage for early stage RCC was relatively poor, while combined with USP39 expression could significantly predict the survival and prognosis of RCC patients. In addition, knockdown of USP39 could significantly inhibit the malignant proliferation, cell colony formation and cell cycle blockage of RCC 786-O and ACHN cells, suggesting that USP39 is an important pro-oncogene in RCC, which is consistent with the study of Xu et al [22].

Targeted therapy plays an anti-tumor role by reducing the size of primary tumors and metastatic sites. Among several targeted therapies, the research on VEGF-A antibodies has become a breakthrough in the treatment of patients with metastatic RCC, such as bevacizumab, which brings new hope for the limited efficacy of TKIs [42]. VEGF-A belongs to the family of platelet-derived growth factors, which is a critical endothelial cell-specific mitogen and vascular permeability inducing factor, stimulating tumor angiogenesis [43]. High expression of VEGF-A mRNA could be detected in almost all malignant tumors [44]. Clinical studies showed that individuals with highly expressed VEGF-A were associated with an increased RCC risk $[45,46]$. A previous investigation demonstrated that RCC patients with VEGF-A-2578 genotype had poor prognosis, including a higher death risk, a larger tumor size, and a worse tumor grade, compared with patients carrying other genotypes [47]. In a study exploring the correlation between angiogenetic markers and RCC outcomes, VEGF-A was found positive in more tumors of immunohistochemistry results [48]. The VEGF-A gene contains eight exons, which are not present in the one VEGF-A at the same time [28]. Instead, the eight exons are recombined by alternative splicing of premRNA to produce different VEGF-A subtypes, which determine their structure, function and affinity to the receptor [49]. Like previous analysis, traditional VEGF-A subtypes are angiogenic, including exon 1-5, 6a, 6b, 7a, 7b and 8a, which are usually identified as VEGF-Axxx, xxx for the number of amino acids [50]. In 2002 and 2004, Bates et al identified another VEGF-Axxx subtype, whose C-terminal exon formed an alternative open reading frame containing 6 amino acids because of distal splicing, usually called VEGF$\operatorname{Axxxb}[28,51]$. The pro-angiogenic VEGF-A165a would be overexpressed and predominated over the VEGF-A165b under cancer conditions. VEGF-Axxx usually promotes angiogenesis, while VEGF-Axxxb is 
just the opposite $[52,53]$. Although the mechanism of VEGF-Axxxb inhibiting angiogenesis has not been elucidated, studies in recent years have revealed that VEGF-A165b can bind to VEGFR-1 and VEGFR-2, but only slightly initiate receptor signal to induce tyrosine phosphorylation, thus reducing angiogenesis [26, 54]. Therefore, some studies reported that the VEGF-A level was not related to RCC risk or outcomes, which potentially resulted from the role of VEGF-Axxxb [55].

SRSFs are involved in splicing regulation and promoting U1 and U2 snRNP binding to splicing sites, whose activities are influenced by SRPKs [56, 57]. SRPK1 is the first identified protein kinase of SR [58]. SRPK1 and other SR protein kinases can phosphorylate SR proteins to facilitate spliceosome assembly [59]. The splicing of VEGF-A exon 8 has been widely studied, and several pathways are found to be associated with VEGF-Axxxb formation. On one hand, growth factors stimulate SRSF1 phosphorylation via SRPK1, thereby allowing SRSF1 to be transported to the nucleus and bind to the proximal splicing site, leading to VEGF-Axxxa isoform formation [60]. On the other hand, downregulation of SRSF1 and SRPK1 switches the splicing of VEGF-A mRNA to generate more VEGF-Axxxb [32]. Upregulation of SRPK1 could lead to the pro-angiogenic isoform of VEGF overexpression and promote disease progression, resulting in poor outcomes[30,61]. Tumor cells attempt to kidnap VEGF-A165a and expel VEGFA-165b so that they could promote cancer development [60]. This critical process might arise form VEGF splicing regulation. And the inhibition of SRPK1 has been demonstrated to attenuate angiogenesis by altering VEGFA-165a to VEGFA-165b in cancer and kidney-related study [32]. Beatrice et al [62] reported that SRPK1 directly acted on SRSF1 to promote VEGF-A splicing to form VEGF-A165 and VEGF-A165b isomers in renal epithelial cells and colon cancer cells, and down-regulation of SRPK1 increased VEGF-A165b expression, while the selective SRPK1 inhibitors brought the anti-angiogenesis effect. In addition, SRPK1 binding SRSF1 has a positive effect on VEGF-A165a alternative splicing and a negative effect on VEGFA165b alternative splicing [63]. All these findings indicate a potential disease therapy, such as SRPKs or SRSFs inhibitor and their interaction blockers [64]. In our study, we also demonstrated that SRPK1 and SRSF1 were involved in the production of alternative splicing of VEGF-A165b in RCC cells and promoted cancer progression, which is consistent with the anti-angiogenic effect of VEGF-A165b in previous studies [52-54].

USP39 is a member of the de-ubiquitin enzyme family, and the SR-related protein 65KD participates in splice assembly without protease activity or ubiquitin activity [10], suggesting that USP39 may act as a mRNA splicing factor. Makarova et al found that USP39 played a regulatory role in pre-mRNA maturation through U4/U6. U5 tri-snRNP and ZnF-UBP domains of USP39 were also found to be the key regions for recruiting and / or activating splice complexes $[10,65]$. USP39 can promote the splicing maturation and normal function of oncogenes mRNA such as Aurora-B,RB1 and mdm-x by regulating splicing complex $[12,66,67]$. Interestingly, co-immunoprecipitation and mass spectrometry analysis in our study also revealed that USP39 could interact with SRPK1 and SRSF1 in RCC cells and participated in VEGFA mRNA splicing. In addition, USP39 overexpression could promote SRPK1 phosphorylation of SRSF1, while knockdown of USP39 worked oppositely, confirming that USP39 and SRPK1 play a role through direct binding rather than affecting their transcription or translation. This also suggests that USP39 has a negative effect on VEGF-A165b alternative spliceosome, which fits the effect of SRPK1 on VEGF-A165b. 
Uniquely, USP39 bond with SRPK1 through fragments (101-565) to promote the phosphorylation and interaction of SRSF1 by SRPK1. USPs are usually expected to be formed by an inactive ubiquitin-specific protease (iUSP) domain and a zinc finger ubiquitin binding domain (ZnF-UBP) [35, 65]. The ZnF of USP39 domain participates in the activities of neighboring domains dependent on ubiquitin but cannot bind ubiquitin itself because of the lack of zinc-binding sites [68]. This suggests that the biological role in mRNA alternative splicing requires additional mediation. A previous study reported that the RS-like domain (AA 1-100) was the only binding truncation of SUMOylation instead of other domains crossing AA 101-565 (ZnF domain, UCH1 domain, or UCH2 domain) in prostate cancer [35]. Previous concepts tend to believe that the $\mathrm{N}$-terminal domain, like RS domains of SR proteins, has rich arginine/serine/glutamate, which is critical for recruiting TRI-snRNP into the pre-spliceosome [69]. Interestingly, our results showed the $\mathrm{ZnF}$ domain, $\mathrm{UCH} 1$ domain, or $\mathrm{UCH} 2$ domain became the binding site. This potentially originating from the iUSP domain cannot bind ubiquitin either, and the ZnF of USP39 could interact with the splicing independent on ubiquitin [65], which provides the opportunity for SRSF1 binding to USP39(101-565), though further exploration is required to confirm the conclusion.

In summary, we firstly demonstrated the value of USP39 in predicting survival, recurrence and metastasis in RCC patients, especially in those with low TNM stage, and that TNM stage combined with USP39 expression was superior to the single index. We have discovered a new regulatory network among USP39, SRPK1, SRSF1 and VEGF-A165b, which can promote the tumorigenesis and development of RCC. USP39 downregulation could inhibit RCC cell proliferation and progression, suggesting that USP39 may prove to be a potential target for inhibiting RCC. These findings may provide a theoretical basis for the development of new targets for the treatment of RCC.

\section{Declarations}

\section{Author Contributions}

Xin-gang Cui had full access to all the data in the study and takes responsibility for the integrity of the data and the accuracy of the data analysis. Study concept and design: Cui, Shi, Song, Pan. Acquisition of data: Xu, W. Chen, Zhou. Analysis and interpretation of data: Pan, Xu, W. Chen. Drafting of the manuscript: Pan, Xu, W. Chen. Critical revision of the manuscript for important intellectual content: Cui, Shi, Pan. Statistical analysis: J. Chen, Ye, Gan. Obtaining funding: Cui, Pan. Administrative, technical, or material support: Pan, Xu, W. Chen. Supervision: Cui, Shi, Song, Pan.

\section{Declaration of Competing Interests}

The authors declare no conflicts of interest.

\section{Acknowledgments}

We express sincere thanks to $Y$ Wu and the Key Laboratory of Cell Differentiation and Apoptosis of the National Ministry of Education (Shanghai Jiaotong University School of Medicine, Shanghai, China) for 
their USP39 fragment plasmids. We also thanks to Prof. Shunxing Zhang (The Faculty of Medical English, Second Military Medical University) for the English language editing to improve the expression.

\section{Data Sharing Statement}

The data that support the findings of this study are available from the corresponding author upon reasonable request.

\section{Funding}

This work was sponsored by the National Natural Science Foundation of China (No. 81772747, 81974391); the National Natural Science Foundation of China for Youths (No. 81702515); the Program of Shanghai Academic/Technology Research Leader (No. 19XD1405100); Shanghai Sailing Program (No. 17YF1425400); Shanghai "Rising Stars of Medical Talent" Youth Development Program: Outstanding Youth Medical Talents (X. Cui) and Youth Medical Talents - Specialist Program (X. Pan); the Technology Project of Jiading District Health System (No.2019-QN-03, JDKW-2017-W13); Project of Health and Family Planning Commission of Shanghai (No.20174Y0175); Key Discipline Construction Project of Pudong Health and Family Planning Commission of Shanghai (No.PWZxk2017-19).

\section{References}

1. RL S, KD M, A J. Cancer statistics, 2020. CA: a cancer journal for clinicians. 2020; 70: 7-30.

2. U C, K B, A B, SA B, F B, J C, et al. Epidemiology of Renal Cell Carcinoma. European urology. 2019; 75 : 74-84.

3. SI T, S P, I K, I A, I A, KG S, et al. Implementation and external validation of Preoperative Aspects and Dimensions Used for an Anatomical (PADUA) score for predicting complications in 74 consecutive partial nephrectomies. BJU international. 2012; 109: 1813-1818.

4. PC B, BI R. Treatment of renal cell carcinoma: Current status and future directions. CA: a cancer journal for clinicians. 2017; 67: 507-524.

5. Iacovelli R, Alesini D, Palazzo A, Trenta P, Santoni M, De Marchis L, et al. Targeted therapies and complete responses in first line treatment of metastatic renal cell carcinoma. A meta-analysis of published trials. Cancer Treat Rev. 2014; 40: 271-275.

6. RR M, D B, TK C. Evolving Systemic Treatment Landscape for Patients With Advanced Renal Cell Carcinoma. Journal of clinical oncology : official journal of the American Society of Clinical Oncology. 2018: JCO2018790253.

7. EM P, S L, RA F. Targeted therapies for renal cell carcinoma. Nature reviews Nephrology. 2017; 13 : 496-511.

8. Climente-González H, Porta-Pardo E, Godzik A, Eyras E. The Functional Impact of Alternative Splicing in Cancer. Cell Rep. 2017; 20: 2215-2226. 
9. Dvinge H, Guenthoer J, Porter PL, Bradley RK. RNA components of the spliceosome regulate tissueand cancer-specific alternative splicing. Genome Res. 2019; 29: 1591-1604.

10. OV M, EM M, R L. The 65 and $110 \mathrm{kDa}$ SR-related proteins of the U4/U6.U5 tri-snRNP are essential for the assembly of mature spliceosomes. The EMBO journal. 2001; 20: 2553-2563.

11. Lygerou Z, Christophides G, Séraphin B. A novel genetic screen for snRNP assembly factors in yeast identifies a conserved protein, Sad1p, also required for pre-mRNA splicing. Mol Cell Biol. 1999; 19: 2008-2020.

12. van Leuken RJ, Luna-Vargas MP, Sixma TK, Wolthuis RM, Medema RH. Usp39 is essential for mitotic spindle checkpoint integrity and controls mRNA-levels of aurora B. Cell Cycle. 2008; 7: 2710-2719.

13. Ye Y, Scheel H, Hofmann K, Komander D. Dissection of USP catalytic domains reveals five common insertion points. Mol Biosyst. 2009; 5: 1797-1808.

14. Yuan X, Sun X, Shi X, Jiang C, Yu D, Zhang W, et al. USP39 promotes the growth of human hepatocellular carcinoma in vitro and in vivo. Oncol Rep. 2015; 34: 823-832.

15. An Y, Yang S, Guo K, Ma B, Wang Y. Reduced USP39 expression inhibits malignant proliferation of medullary thyroid carcinoma in vitro. World J Surg Oncol. 2015; 13: 255.

16. Huang Y, Pan X-W, Li L, Chen L, Liu X, Lu J-L, et al. Overexpression of USP39 predicts poor prognosis and promotes tumorigenesis of prostate cancer via promoting EGFR mRNA maturation and transcription elongation. Oncotarget. 2016; 7: 22016-22030.

17. Wang X, Yu Q, Huang L, Yu P. Lentivirus-mediated inhibition of USP39 suppresses the growth of gastric cancer cells via PARP activation. Molecular medicine reports. 2016; 14: 301-306.

18. Gan Z, Han K, Lin S, Hu H, Shen Z, Min D. Knockdown of ubiquitin-specific peptidase 39 inhibited the growth of osteosarcoma cells and induced apoptosis in vitro. Biol Res. 2017; 50: 15.

19. Lin Z, Xiong L, Lin Q. Ubiquitin-specific protease 39 is overexpressed in human lung cancer and promotes tumor cell proliferation in vitro. Mol Cell Biochem. 2016; 422.

20. Ding K, Ji J, Zhang X, Huang B, Chen A, Zhang D, et al. RNA splicing factor USP39 promotes glioma progression by inducing TAZ mRNA maturation. Oncogene. 2019; 38: 6414-28.

21. Wang H, Ji X, Liu X, Yao R, Chi J, Liu S, et al. Lentivirus-mediated inhibition of USP39 suppresses the growth of breast cancer cells in vitro. Oncol Rep. 2013; 30: 2871-2877.

22. Xu Y, Zhu MR, Zhang JY, Si GM, Lv JJ. Knockdown of ubiquitinspecific peptidase 39 inhibits the malignant progression of human renal cell carcinoma. Mol Med Rep. 2018; 17: 4729-4235.

23. X L, X S, P C. Hallmarks of Endothelial Cell Metabolism in Health and Disease. Cell metabolism. 2019; 30: 414-433.

24. RS A, DS C, N F. VEGF in Signaling and Disease: Beyond Discovery and Development. Cell. 2019; 176: 1248-1264.

25. Perrin RM, Konopatskaya O, Qiu Y, Harper S, Bates DO, Churchill AJ. Diabetic retinopathy is associated with a switch in splicing from anti- to pro-angiogenic isoforms of vascular endothelial growth factor. Diabetologia. 2005; 48: 2422-2427. 
26. Woolard J, Wang W-Y, Bevan HS, Qiu Y, Morbidelli L, Pritchard-Jones RO, et al. VEGF165b, an inhibitory vascular endothelial growth factor splice variant: mechanism of action, in vivo effect on angiogenesis and endogenous protein expression. Cancer Res. 2004; 64: 7822-7835.

27. Pritchard-Jones RO, Dunn DBA, Qiu Y, Varey AHR, Orlando A, Rigby $H$, et al. Expression of VEGF(xxx)b, the inhibitory isoforms of VEGF, in malignant melanoma. Br J Cancer. 2007; 97: 223-230.

28. Bates DO, Cui T-G, Doughty JM, Winkler M, Sugiono M, Shields JD, et al. VEGF165b, an inhibitory splice variant of vascular endothelial growth factor, is down-regulated in renal cell carcinoma. Cancer Res. 2002; 62: 4123-4131.

29. L X, C C, C W, LM Z. Association between bevacizumab-related chemotherapy regimens and serum vascular endothelial growth factor-A165b level in patients with metastatic colorectal cancer. European review for medical and pharmacological sciences. 2014; 18: 2687-2692.

30. Gammons MV, Lucas R, Dean R, Coupland SE, Oltean S, Bates DO. Targeting SRPK1 to control VEGFmediated tumour angiogenesis in metastatic melanoma. Br J Cancer. 2014; 111: 477-485.

31. Sargent KM, Clopton DT, Lu N, Pohlmeier WE, Cupp AS. VEGFA splicing: divergent isoforms regulate spermatogonial stem cell maintenance. Cell Tissue Res. 2016; 363: 31-45.

32. EM A, S O, J H, MV G, M H-Z, GI W, et al. WT1 mutants reveal SRPK1 to be a downstream angiogenesis target by altering VEGF splicing. Cancer cell. 2011; 20: 768-780.

33. Gumz ML, Zou H, Kreinest PA, Childs AC, Belmonte LS, LeGrand SN, et al. Secreted frizzled-related protein 1 loss contributes to tumor phenotype of clear cell renal cell carcinoma. Clinical cancer research : an official journal of the American Association for Cancer Research. 2007; 13: 4740-4749.

34. Zhao H, Ljungberg B, Grankvist K, Rasmuson T, Tibshirani R, Brooks JD. Gene expression profiling predicts survival in conventional renal cell carcinoma. PLoS Med. 2006; 3: e13.

35. Wen D, Xu Z, Xia L, Liu X, Tu Y, Lei H, et al. Important role of SUMOylation of Spliceosome factors in prostate cancer cells. J Proteome Res. 2014; 13: 3571-3582.

36. JA G, BI R. Recent progress in the management of advanced renal cell carcinoma. CA: a cancer journal for clinicians. 2007; 57: 112-125.

37. Ghosh G, Adams JA. Phosphorylation mechanism and structure of serine-arginine protein kinases. FEBS J. 2011; 278: 587-597.

38. L L, W Z, X Q, H L, J Y, S W, et al. Randomized study of autologous cytokine-induced killer cell immunotherapy in metastatic renal carcinoma. Clinical cancer research : an official journal of the American Association for Cancer Research. 2012; 18: 1751-1759.

39. RJ A, RE H, HL K, JA T, P T, C S, et al. Vaccination of metastatic renal cancer patients with MVA-5T4: a randomized, double-blind, placebo-controlled phase III study. Clinical cancer research : an official journal of the American Association for Cancer Research. 2010; 16: 5539-47.

40. Cairns P. Renal cell carcinoma. Cancer Biomark. 2010; 9: 461-473.

41. Capitanio U, Montorsi F. Renal cancer. Lancet. 2016; 387: 894-906. 
42. JJ H, MP P, S S, C S, L A, M S, et al. Renal cell carcinoma. Nature reviews Disease primers. 2017; 3: 17009.

43. Porta C, Paglino C, De Amici M, Quaglini S, Sacchi L, Imarisio I, et al. Predictive value of baseline serum vascular endothelial growth factor and neutrophil gelatinase-associated lipocalin in advanced kidney cancer patients receiving sunitinib. Kidney Int. 2010; 77: 809-815.

44. Ferrara N, Davis-Smyth T. The biology of vascular endothelial growth factor. Endocr Rev. 1997; 18.

45. Ajaz S, Khaliq S, Abid A, Hassan AS, Hashmi A, Sultan G, et al. Association of a single-nucleotide polymorphism in the promoter region of the VEGF gene with the risk of renal cell carcinoma. Genet Test Mol Biomarkers. 2011; 15: 653-657.

46. Bruyère $F$, Hovens $C M$, Marson M-N, d'Arcier BF, Costello AJ, Watier $H$, et al. VEGF polymorphisms are associated with an increasing risk of developing renal cell carcinoma. J Urol. 2010; 184: 1273-1278.

47. Zhong W, Wang X, Pan B, Su Z. Association of vascular endothelial growth factor polymorphisms with clinical outcome of renal cell carcinoma patients. Tumour Biol. 2014; 35: 9839-9845.

48. Stubbs C, Bardoli AD, Afshar M, Pirrie S, Miscoria M, Wheeley I, et al. A Study of Angiogenesis Markers in Patients with Renal Cell Carcinoma Undergoing Therapy with Sunitinib. Anticancer Res. 2017; 37: 253-259.

49. Waltenberger J, Claesson-Welsh L, Siegbahn A, Shibuya M, Heldin $\mathrm{CH}$. Different signal transduction properties of KDR and Flt1, two receptors for vascular endothelial growth factor. J Biol Chem. 1994; 269: 26988-26995.

50. Houck KA, Ferrara N, Winer J, Cachianes G, Li B, Leung DW. The vascular endothelial growth factor family: identification of a fourth molecular species and characterization of alternative splicing of RNA. Mol Endocrinol. 1991; 5: 1806-1814.

51. Perrin RM, Konopatskaya O, Qiu Y, Harper S, Bates DO, Churchill AJ. Diabetic retinopathy is associated with a switch in splicing from anti- to pro-angiogenic isoforms of vascular endothelial growth factor. Diabetologia. 2005; 48: 2422-2427.

52. Carter JG, Gammons MVR, Damodaran G, Churchill AJ, Harper SJ, Bates DO. The carboxyl terminus of VEGF-A is a potential target for anti-angiogenic therapy. Angiogenesis. 2015; 18: 23-30.

53. Hulse RP, Beazley-Long N, Hua J, Kennedy H, Prager J, Bevan H, et al. Regulation of alternative VEGFA mRNA splicing is a therapeutic target for analgesia. Neurobiol Dis. 2014; 71: 245-259.

54. Cébe Suarez S, Pieren M, Cariolato L, Arn S, Hoffmann U, Bogucki A, et al. A VEGF-A splice variant defective for heparan sulfate and neuropilin-1 binding shows attenuated signaling through VEGFR-2. Cell Mol Life Sci. 2006; 63: 2067-2077.

55. Sáenz-López P, Vazquez F, Cozar JM, Carretero R, Garrido F, Ruiz-Cabello F. VEGF polymorphisms are not associated with an increased risk of developing renal cell carcinoma in Spanish population. Hum Immunol. 2013; 74.

56. Zhou Z, Fu X-D. Regulation of splicing by SR proteins and SR protein-specific kinases. Chromosoma. 2013; 122: 191-207. 
57. Howard JM, Sanford JR. The RNAissance family: SR proteins as multifaceted regulators of gene expression. Wiley Interdiscip Rev RNA. 2015; 6.

58. Gui JF, Lane WS, Fu XD. A serine kinase regulates intracellular localization of splicing factors in the cell cycle. Nature. 1994; 369: 678-682.

59. Aubol BE, Wu G, Keshwani MM, Movassat M, Fattet L, Hertel KJ, et al. Release of SR Proteins from CLK1 by SRPK1: A Symbiotic Kinase System for Phosphorylation Control of Pre-mRNA Splicing. Mol Cell. 2016; 63: 218-228.

60. Nowak DG, Amin EM, Rennel ES, Hoareau-Aveilla C, Gammons M, Damodoran G, et al. Regulation of vascular endothelial growth factor (VEGF) splicing from pro-angiogenic to anti-angiogenic isoforms: a novel therapeutic strategy for angiogenesis. J Biol Chem. 2010; 285: 5532-5540.

61. Li X-h, Song J-w, Liu J-I, Wu S, Wang L-s, Gong L-y, et al. Serine-arginine protein kinase 1 is associated with breast cancer progression and poor patient survival. Med Oncol. 2014; 31: 83.

62. Gout S, Brambilla E, Boudria A, Drissi R, Lantuejoul S, Gazzeri S, et al. Abnormal expression of the pre-mRNA splicing regulators SRSF1, SRSF2, SRPK1 and SRPK2 in non small cell lung carcinoma. PLoS One. 2012; 7: e46539.

63. Mavrou A, Brakspear K, Hamdollah-Zadeh M, Damodaran G, Babaei-Jadidi R, Oxley J, et al. Serinearginine protein kinase 1 (SRPK1) inhibition as a potential novel targeted therapeutic strategy in prostate cancer. Oncogene. 2015; 34: 4311-4319.

64. Batson J, Toop HD, Redondo C, Babaei-Jadidi R, Chaikuad A, Wearmouth SF, et al. Development of Potent, Selective SRPK1 Inhibitors as Potential Topical Therapeutics for Neovascular Eye Disease. ACS Chem Biol. 2017; 12: 825-832.

65. Hadjivassiliou H, Rosenberg OS, Guthrie C. The crystal structure of S. cerevisiae Sad1, a catalytically inactive deubiquitinase that is broadly required for pre-mRNA splicing. RNA. 2014; 20: 656-669.

66. Allende-Vega N, Dayal S, Agarwala U, Sparks A, Bourdon JC, Saville MK. p53 is activated in response to disruption of the pre-mRNA splicing machinery. Oncogene. 2013; 32.

67. Ríos Y, Melmed S, Lin S, Liu N-A. Zebrafish usp39 mutation leads to rb1 mRNA splicing defect and pituitary lineage expansion. PLoS Genet. 2011; 7: e1001271.

68. Köhler A, Zimmerman E, Schneider M, Hurt E, Zheng N. Structural basis for assembly and activation of the heterotetrameric SAGA histone H2B deubiquitinase module. Cell. 2010; 141: 606-617.

69. Roscigno RF, Garcia-Blanco MA. SR proteins escort the U4/U6.U5 tri-snRNP to the spliceosome. RNA. 1995; 1: 692-706.

\section{Tables}

Table 1. USP39 expression and patient characteristics of Zhao Renal dataset 


\begin{tabular}{|c|c|c|c|c|}
\hline & \multirow[t]{2}{*}{ No. Pts } & \multicolumn{2}{|l|}{ No. USP39 } & \multirow[t]{2}{*}{$p$ Value } \\
\hline & & Low & High & \\
\hline Overall: n (\%) & $176(100.0)$ & $88(50.0)$ & $88(50.0)$ & \\
\hline Male & $101(57.4)$ & $47(26.7)$ & $54(30.7 \rrbracket$ & $0.286^{a}$ \\
\hline Female & $75(42.6)$ & $41 \rrbracket 23.3 \rrbracket$ & $34 \rrbracket 19.3 \rrbracket$ & \\
\hline Mean patient age, y (range) & $65.2(34-85)$ & $63.7(42-85)$ & $66.6 \bowtie 34-85 \rrbracket$ & $\otimes 0.000^{b}$ \\
\hline WHO performance status: n (\%) & & & & $0.146^{a}$ \\
\hline 0 & $65(36.9)$ & $29 \rrbracket 16.5 \rrbracket$ & $36 \rrbracket 20.5 \rrbracket$ & \\
\hline 1 & 63(35.8) & $35(19.9)$ & $28(15.9)$ & \\
\hline 2 & $37(21.0)$ & $16 \rrbracket 9.1 \rrbracket$ & $21 \otimes 11.9 \rrbracket$ & \\
\hline 3 & $10(5.7)$ & $8 \llbracket 4.5 \rrbracket$ & $2 \otimes 1.1 \rrbracket$ & \\
\hline 4 & $1(0.6)$ & $0 \otimes 0.0 \otimes$ & 1区0.6区 & \\
\hline TNM stage: $\mathrm{n}(\%)$ & & & & $0.919^{a}$ \\
\hline ( & $49(27.8)$ & $25 \rrbracket 14.2 \rrbracket$ & $24 \bigotimes 13.6)$ & \\
\hline ० & $29(16.5)$ & $16(9.1)$ & $13(7.4)$ & \\
\hline ( & $40(22.7)$ & 19ه10.8》 & 21ه11.9凶 & \\
\hline ? & $58(33.0)$ & $28 \rrbracket 15.9 \rrbracket$ & $30 \otimes 17.0 \rrbracket$ & \\
\hline Fuhrman grade: $\mathrm{n}(\%)$ & & & & $0.726^{a}$ \\
\hline 1 & $9(5.1)$ & $6 \rrbracket 3.4 \rrbracket$ & $3 \otimes 1.7 \rrbracket$ & \\
\hline 2 & $34(19.3)$ & $18 \rrbracket 10.2 \rrbracket$ & 16ه9.1区 & \\
\hline 3 & $93(52.8)$ & $45 \rrbracket 25.6 \rrbracket$ & $48 \llbracket 27.3 \rrbracket$ & \\
\hline 4 & $40(22.7)$ & $19 \rrbracket 10.8 \rrbracket$ & $21 \otimes 11.9 \rrbracket$ & \\
\hline Patient outcome: n (\%) & & & & $0.008^{a}$ \\
\hline Died & $111(63.1)$ & $47 \rrbracket 21.6 \rrbracket$ & $64(27.3)$ & \\
\hline Alive & $65(36.9)$ & $41 \rrbracket 4.0 \otimes$ & $24 \bowtie 1.1 \rrbracket$ & \\
\hline median OS, mo (95\% Cl) & $39(33.4-44.6)$ & $59(14.1-103.9)$ & $35(23.0-47.0)$ & $0.006^{c}$ \\
\hline
\end{tabular}

${ }^{a}$ chi-square test; ${ }^{b}$ Wilcoxon rank sum test; ${ }^{C}$ Log Rank test 
Analysis of USP39 in the ONCOMINE gene database. The expression level of USP39 was positively correlated with the survival rate of renal cancer patients

Table 2. Univariate and Multivariate Cox regression analysis of OS of Zhao Renal dataset

\begin{tabular}{|c|c|c|c|c|}
\hline & Univariate COX Regr & ssion & Multivariate Cox Rec & ession \\
\hline & $\mathrm{HR}(95 \% \mathrm{Cl})$ & $p$ Value & $\mathrm{HR}(95 \% \mathrm{Cl})$ & $p$ Value \\
\hline Age $^{*}$, years & & 0.125 & & 0.871 \\
\hline$₫ 67$ & 1.00 (referent) & & 1.00 (referent) & \\
\hline$\geq 67$ & $1.344(0.921-1.959)$ & & $1.033(0.701-1.522)$ & \\
\hline Sex & & 0.736 & & 0.491 \\
\hline Male & 1.00 (referent) & & 1.00 (referent) & \\
\hline Female & $0.937(0.642-1.367)$ & & $1.151(0.771-1.718)$ & \\
\hline TNM stage & & 0.000 & & 0.000 \\
\hline$\nabla+\square$ & 1.00 (referent) & & 1.00 (referent) & \\
\hline$\nabla+\square$ & $4.279(2.771-6.607)$ & & $3.812(2.382-6.101)$ & \\
\hline Fuhrman grade & & 0.013 & & 0.683 \\
\hline $1+2$ & 1.00 (referent) & & 1.00 (referent) & \\
\hline $3+4$ & $1.866(1.138-3.060)$ & & $1.114(0.664-1.868)$ & \\
\hline WHO performance status & & 0.000 & & 0.000 \\
\hline 0 & 1.00 (referent) & & 1.00 (referent) & \\
\hline 1 or Greater & $2.460(1.608-3.763)$ & & $2.216(1.419-3.460)$ & \\
\hline USP39 expression & & 0.007 & & 0.014 \\
\hline Low & 1.00 (referent) & & 1.00 (referent) & \\
\hline High & $1.680(1.150-2.455)$ & & $1.629(1.103-2.405)$ & \\
\hline
\end{tabular}

*Divided at median.

Univariate and multivariate analyses showed that the level of USP39 expression was an independent risk factor for overall survival in renal cancer patients. OS: overall survival. 
Figures

A
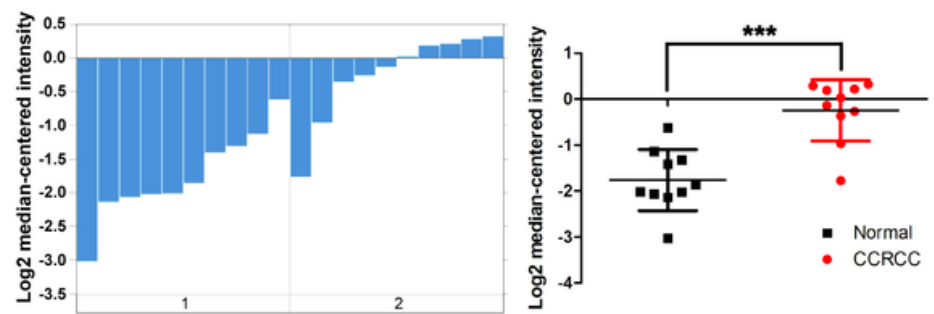

D

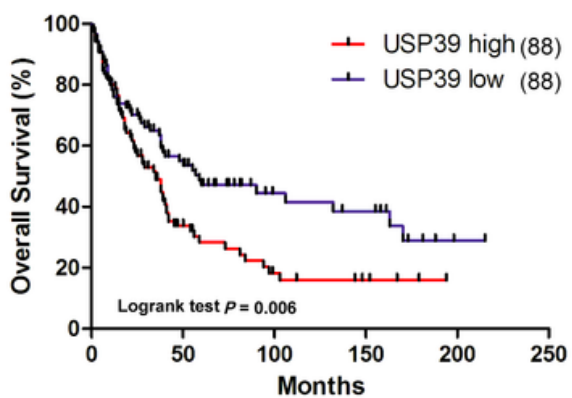

$\mathbf{F}$

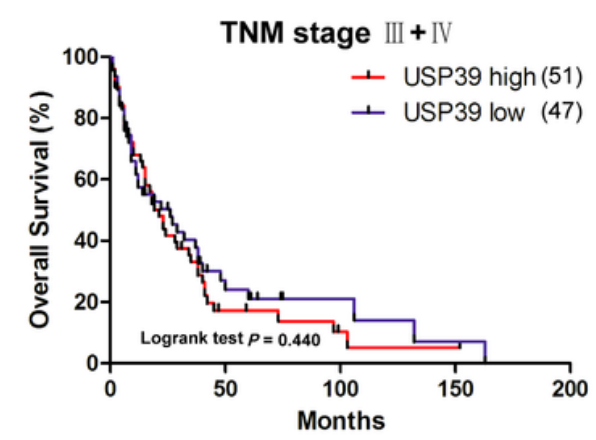

B
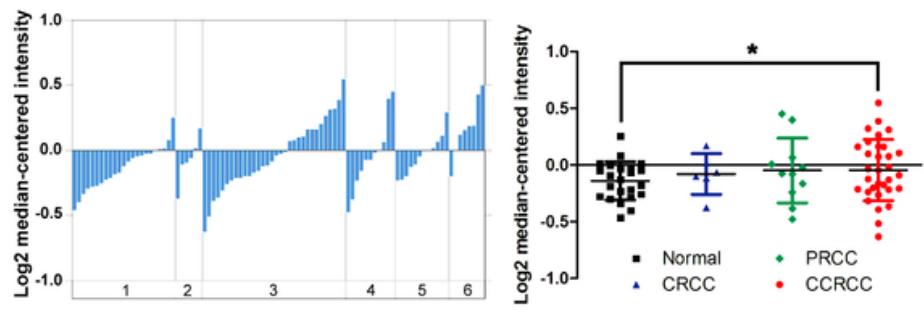

$\mathbf{E}$
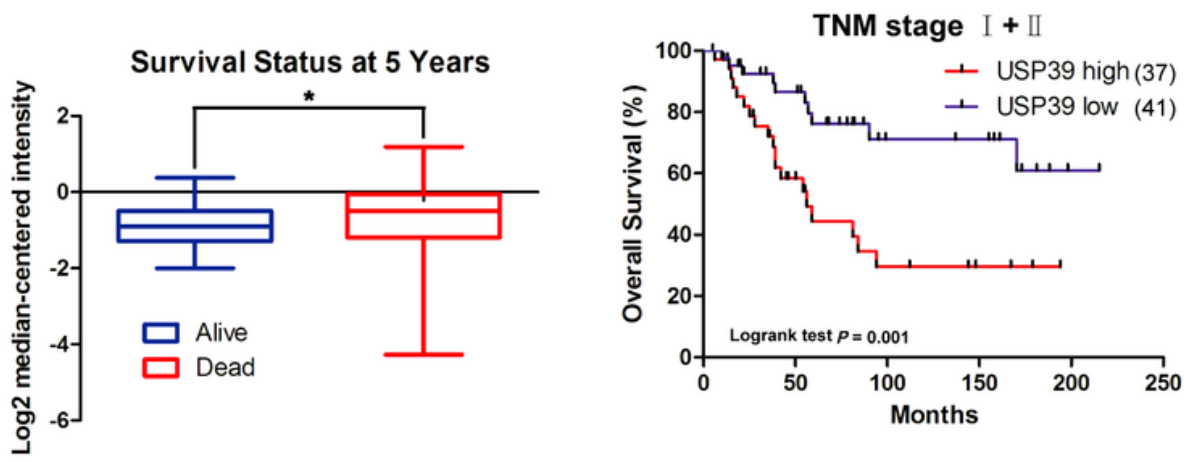

H

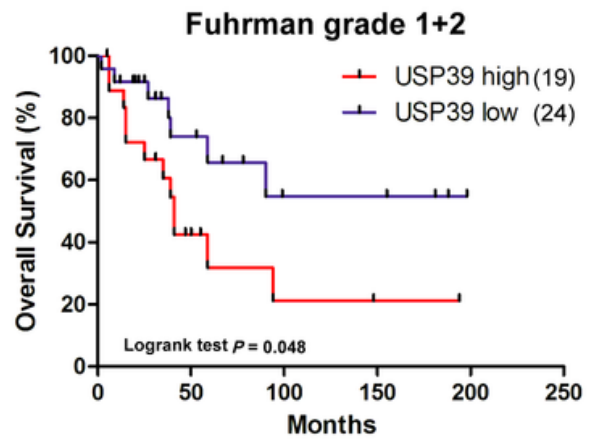

Fuhrman grade $3+4$

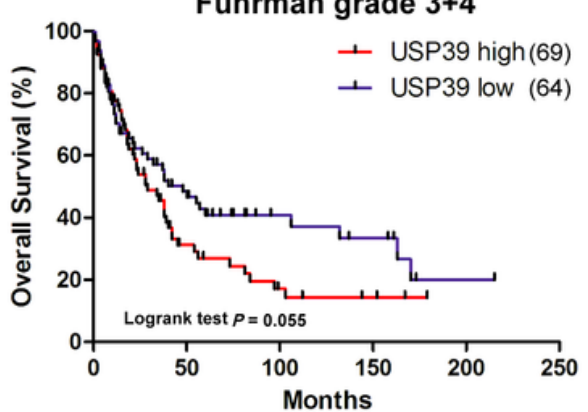

Figure 1

The value of USP39 expression in renal cell carcinoma by ONCOMINE gene database analysis. (A) The expression of USP39 in renal clear cell carcinoma was significantly higher than that in normal renal tissue. (B) USP39 expression was associated with the malignant degree of kidney cancer. (C) Patients with high expression of USP39 had poor survival and prognosis. (D) The expression level of USP39 in patients who died at 5 years and those who survived. $(E, F)$ The overall survival of patients with high or low expression of USP39 in different TNM stages. $(G, H)$ Overall survival of patients with high or low expression of USP39 in different Fuhrman grades. CCRCC: renal clear cell carcinoma; CRCC: color renal cell carcinoma; PRCC: papillary renal cell carcinoma. *, $\mathrm{P}<0.05$; $\star \star \star, ~ P<0.001$ versus normal renal tissue. ${ }^{*} \mathrm{P}<0.05$ versus deaths at 5 years. 


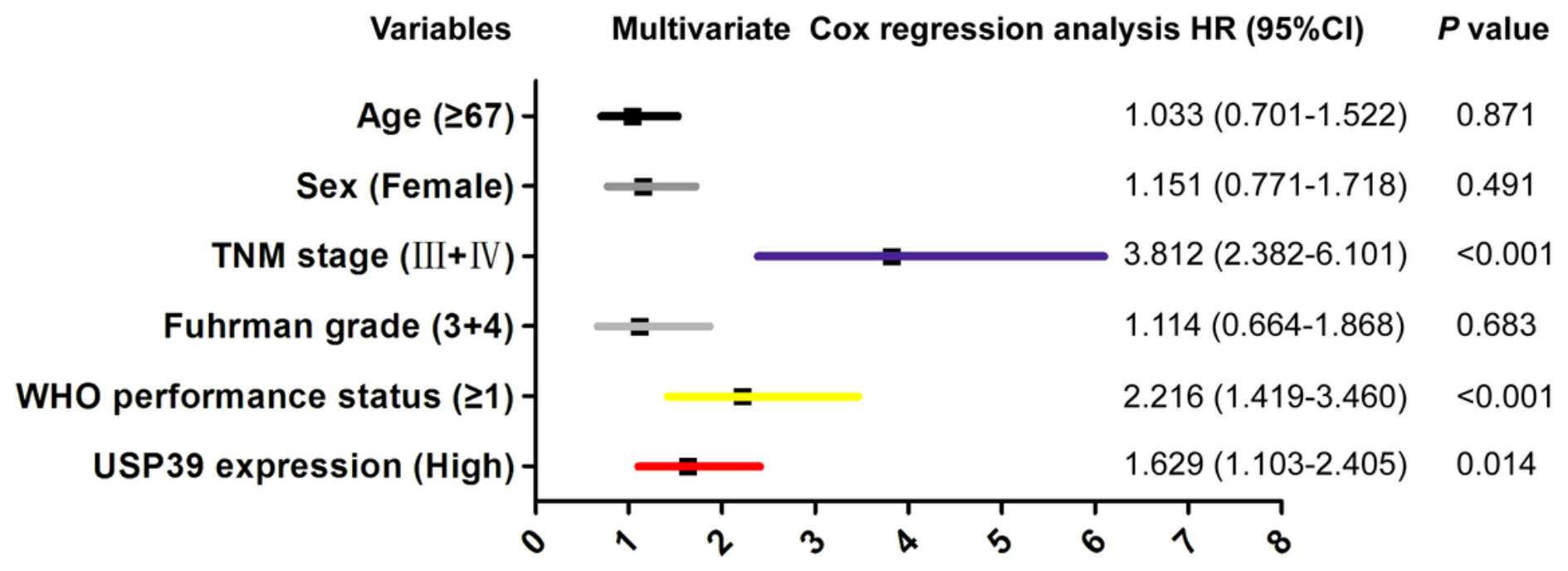

B
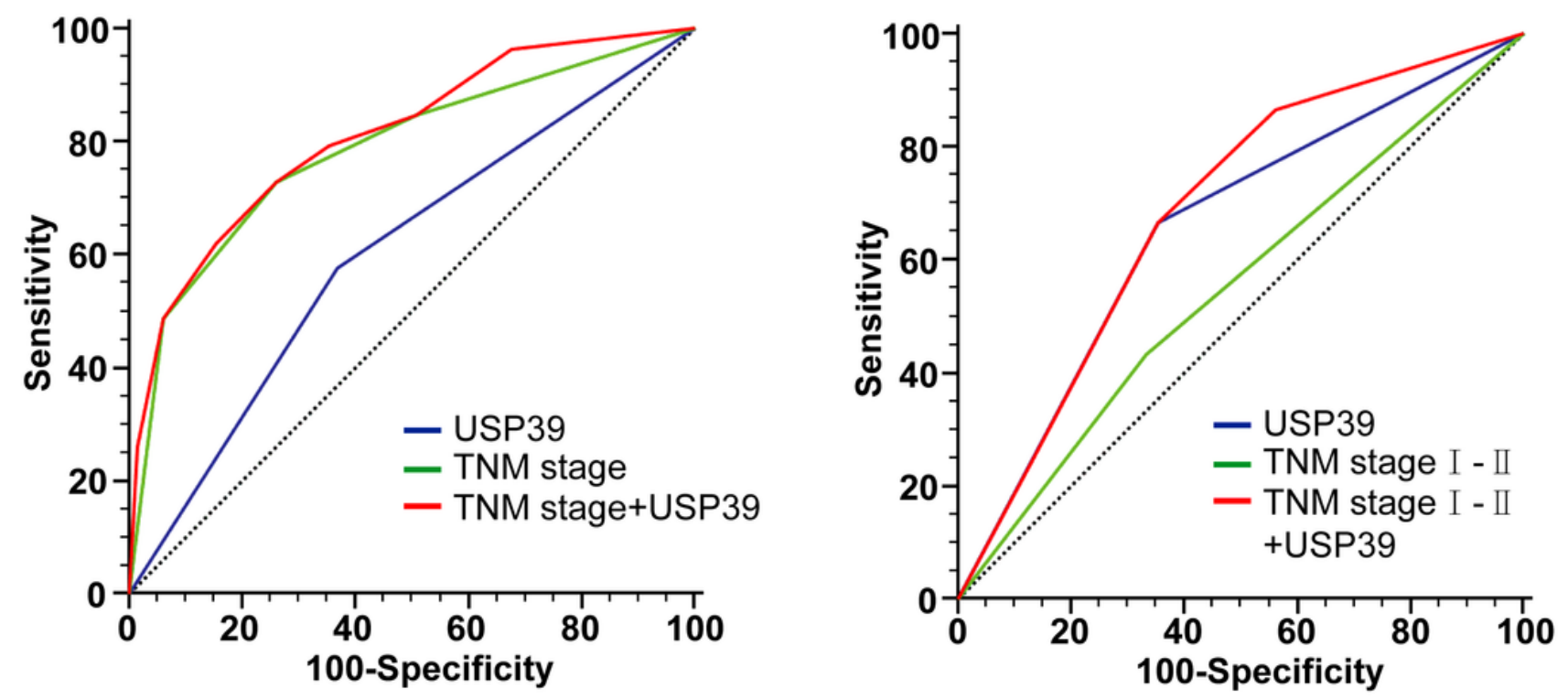

\begin{tabular}{lccc} 
& AUC & $95 \% \mathrm{Cl}$ & $P^{\mathrm{a}}$ \\
\hline TNM Stage+ USP39 & 0.810 & 0.744 to 0865 & \\
TNM Stage & 0.785 & 0.717 to 0.843 & 0.0042 \\
USP39 expression & 0.604 & 0.527 to 0.676 & $<0.0001$ \\
\hline
\end{tabular}

\begin{tabular}{lccc} 
& AUC & $95 \% \mathrm{Cl}$ & $\boldsymbol{P}^{\mathrm{b}}$ \\
\hline TNM Stage I - II + USP39 & 0.686 & 0.571 to 0.786 & 0.0016 \\
USP39 expression & 0.656 & 0.540 to 0.760 & 0.0052 \\
TNM Stage I - II & 0.550 & 0.433 to 0.663 & 0.3840 \\
\hline
\end{tabular}

Figure 2

The expression of TNM combined with USP39 in patients with low stage can significantly predict the survival and prognosis of patients. (A) The level of USP39 expression is an independent risk factor for overall survival in renal cancer patients. (B) ROC curve analysis for predictive the ability of single indicator TNM, single indicator TNM and combined TNM and USP39. (C) For patients with low TNM stage, ROC curve analysis for the predictive ability of the expression level of USP39, TNM stage, and combined indicators. 
A

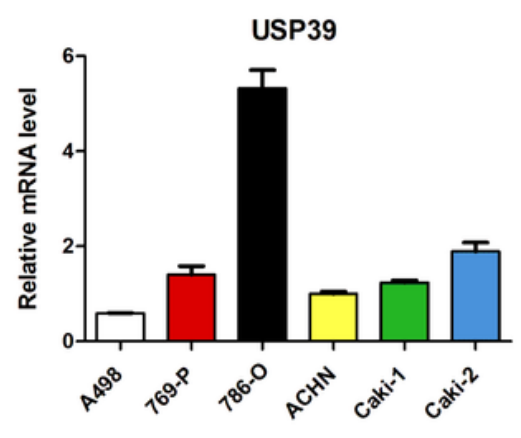

D

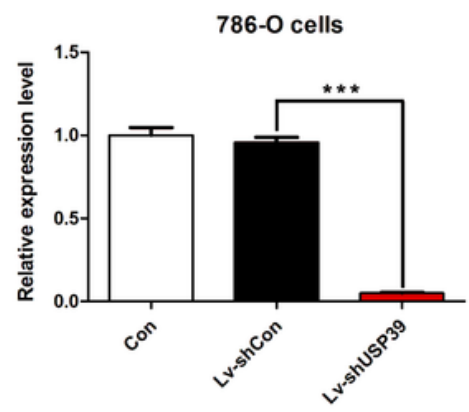

F

$786-0$ cells

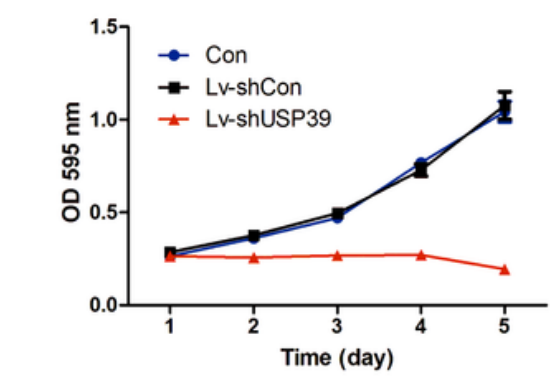

G

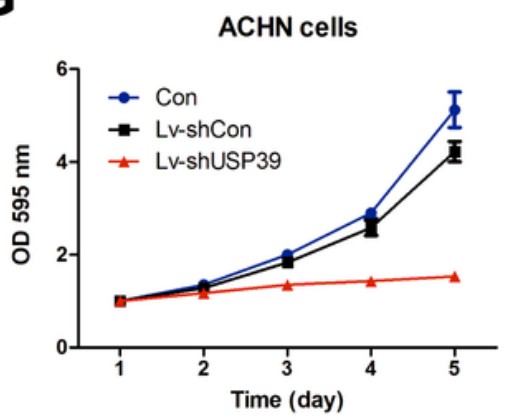

B

C
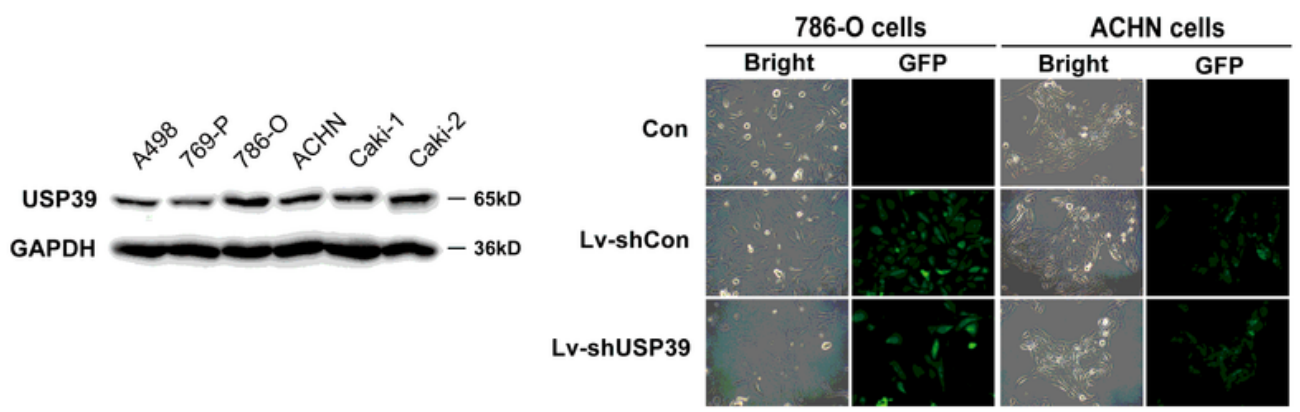

E
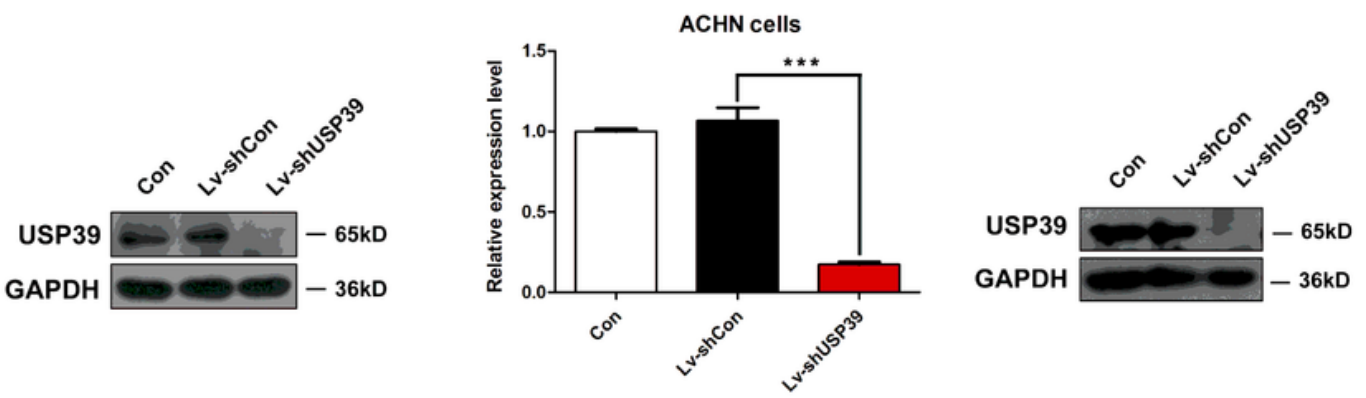

H

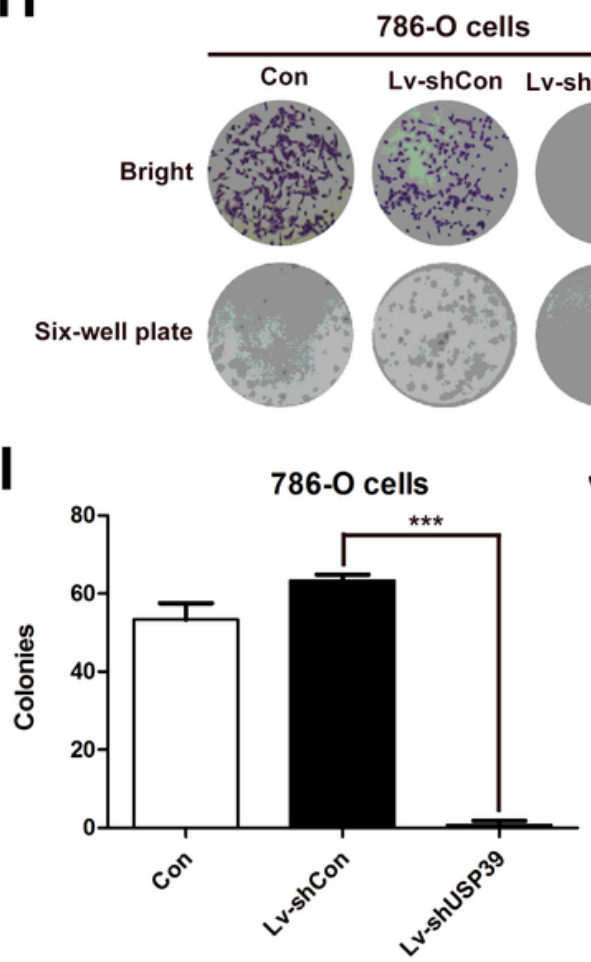

ACHN cells

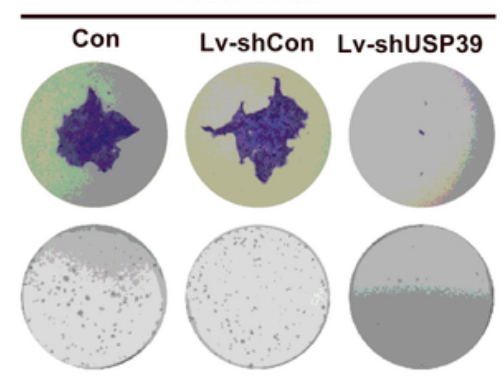

ACHN cells

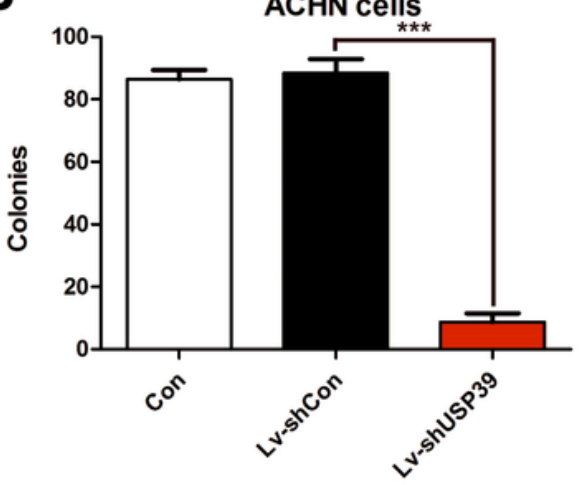

Figure 3

786-o and ACHN cell proliferation after USP39 knockdown. (A,B) The expression level of USP39 in renal cell carcinoma cell lines A498, 769P, 786-0, ACHN, Caki-1 and Caki-2. (C) Fluorescence expression of 786$O$ and $A C H N$ cells after lentivirus infection. $(D, E)$ The expression of USP39 after lentivirus infection as shown by Western blot assay and Real-time fluorescence quantitative PCR. (F,G) MTT growth curves of 
786-o and ACHN cells after USP39 knockdown. $(\mathrm{H}, \mathrm{I}, \mathrm{J})$ The cloning ability of 786-o and ACHN cells after USP39 knockdown. *** $\mathrm{P}<0.001$ versus $\mathrm{Lv}+\mathrm{shCon}$.

A

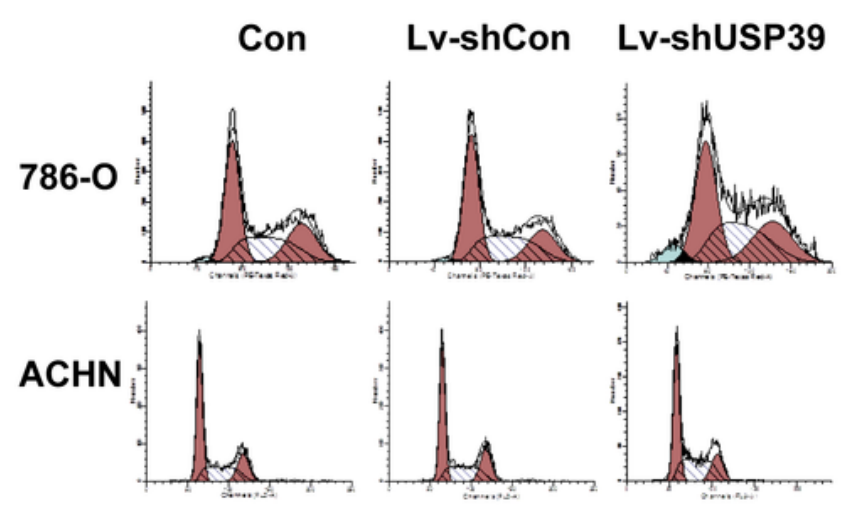

D

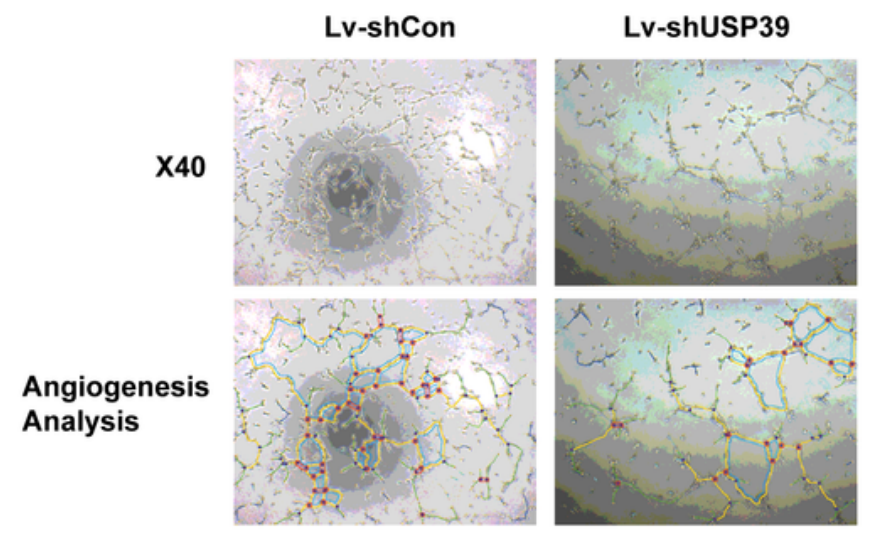

$\mathbf{E}$
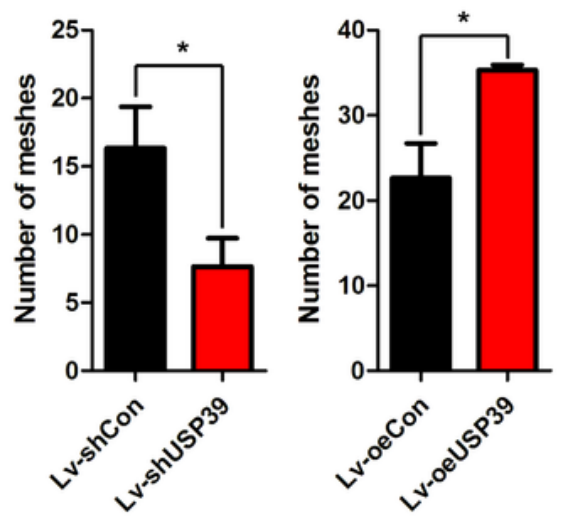

$\mathbf{F}$

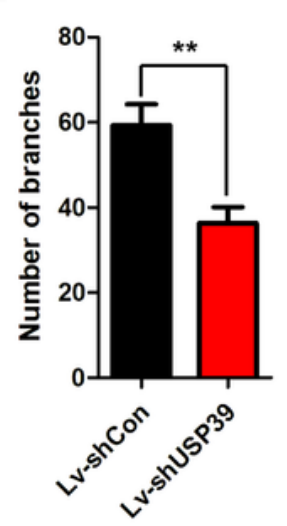

B

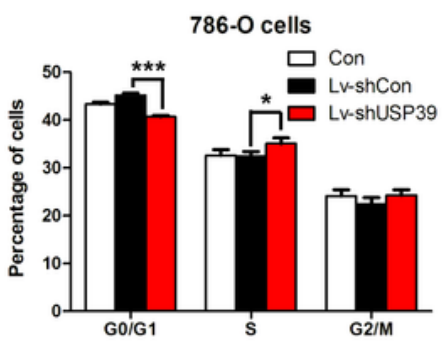

C

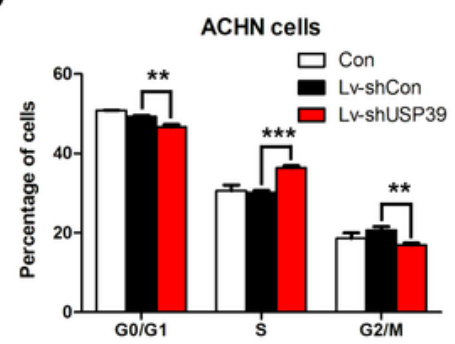

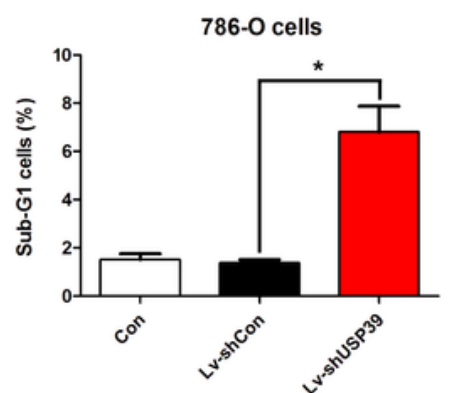

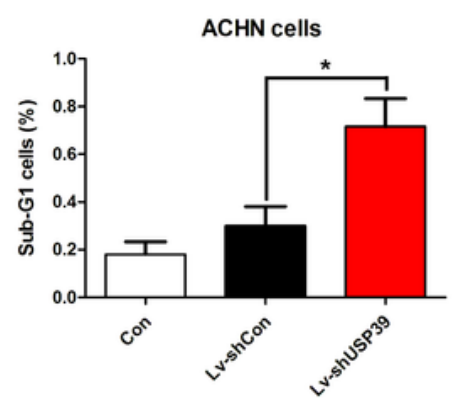

Lv-oeUSP39
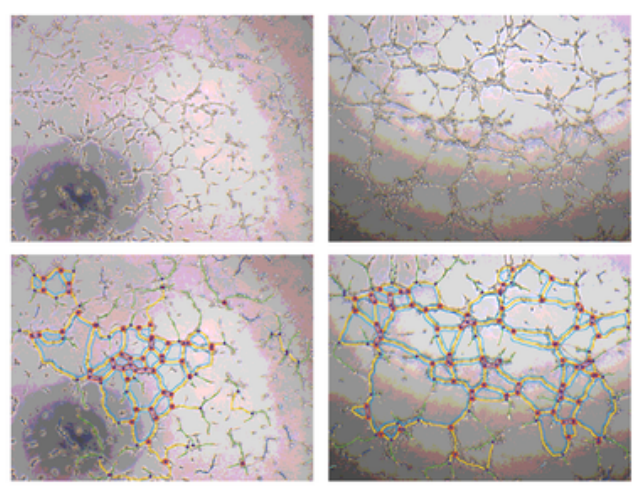

G

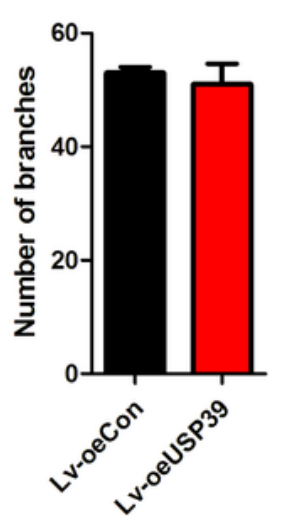

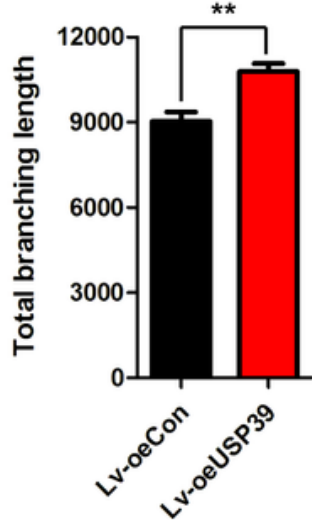

Figure 4

Changes in cell cycle of 786-o and ACHN cells after USP39 knockdown and the effect of USP39 on angiogenesis . (A) The S-phase peaks and subG1 phase peaks of 786-o and ACHN cells were significantly upregulated. $(B, C)$ The proportion of S-phase cells, subG1 phase cells and G0/G1 phase cells in 786-0 
and ACHN cells after USP39 knockdown. (D) Tubular formation and analysis after USP39 knockdown or overexpression. (E, F, G) Tubular formation and tubule branching analysis of HUEVs after USP39 knockdown or overexpression. ${ }^{*} P<0.05 ;{ }^{*} P<0.01$ versus $L v+$ shCon or $L v+o e C o n .{ }^{*} P<0.05,{ }^{*} P<0.01$, *** $\mathrm{P}<0.001$ versus $\mathrm{Lv}+\mathrm{shCon}$.

A

\section{B}

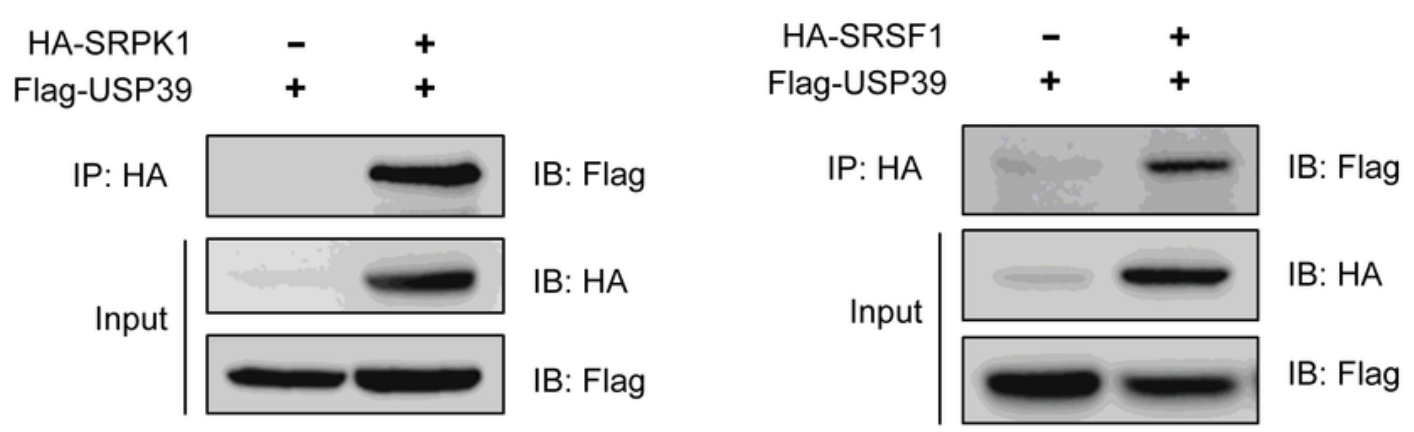

C

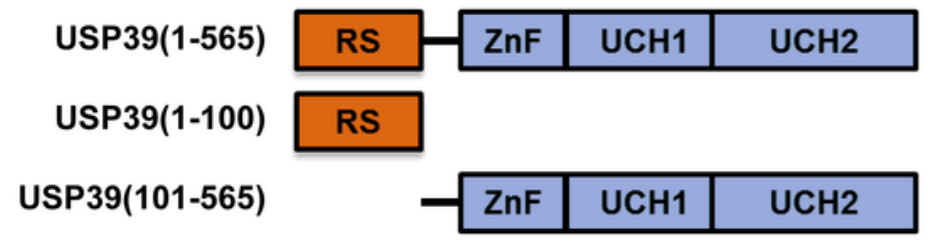

D

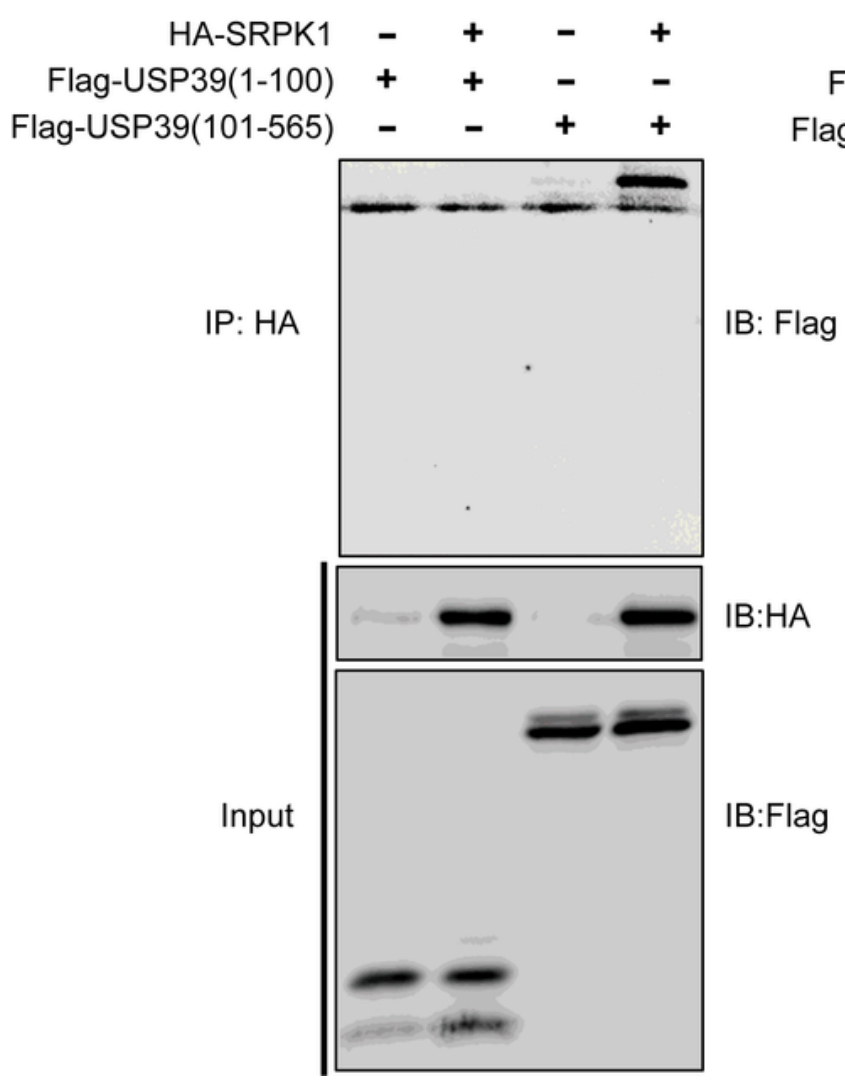

E

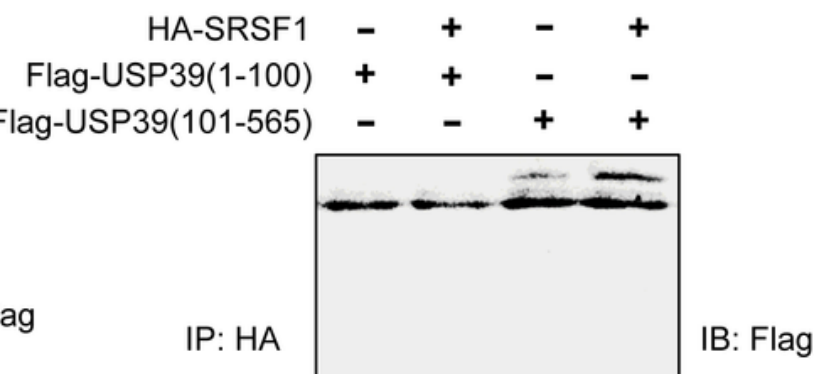

nput

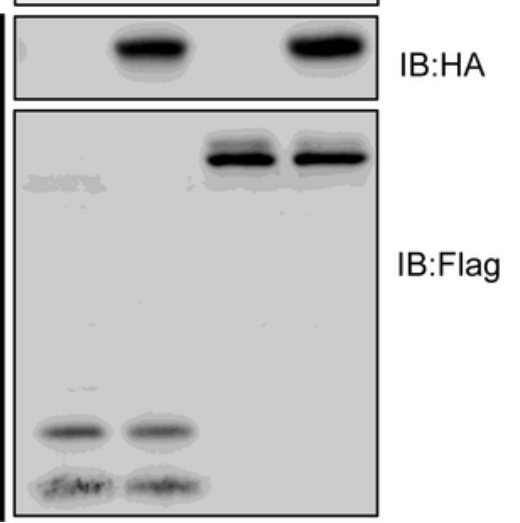

Figure 5 
Mass spectrometry of interaction between USP39 and SRSF1 and SRPK1. (A, B) The plasmids were transfected into 293T cells, and the interaction was verified in pairs. (C, D, E) USP39 fragment plasmids were constructed, and both SRPK1 and SRSF1 interacted with the (101-565) fragment of USP39.

A

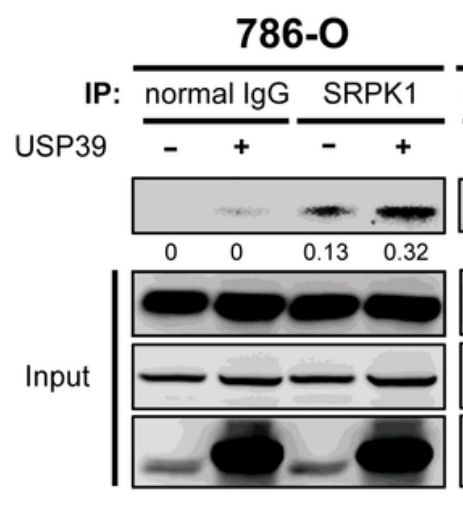

ACHN

C

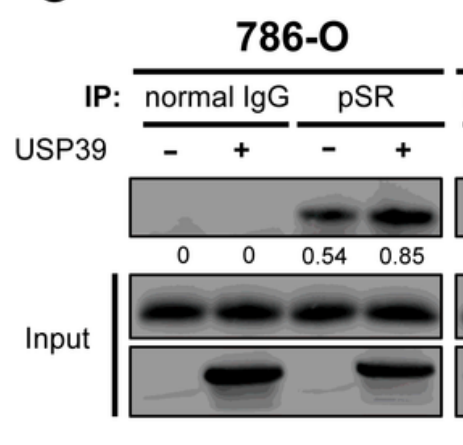

ACHN $\frac{\overline{\text { normal } \lg G}}{-+\quad \mathrm{pSR}}$

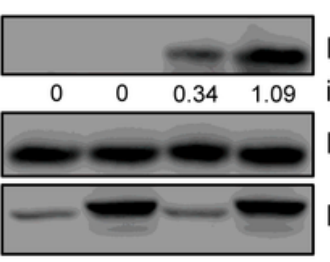

B

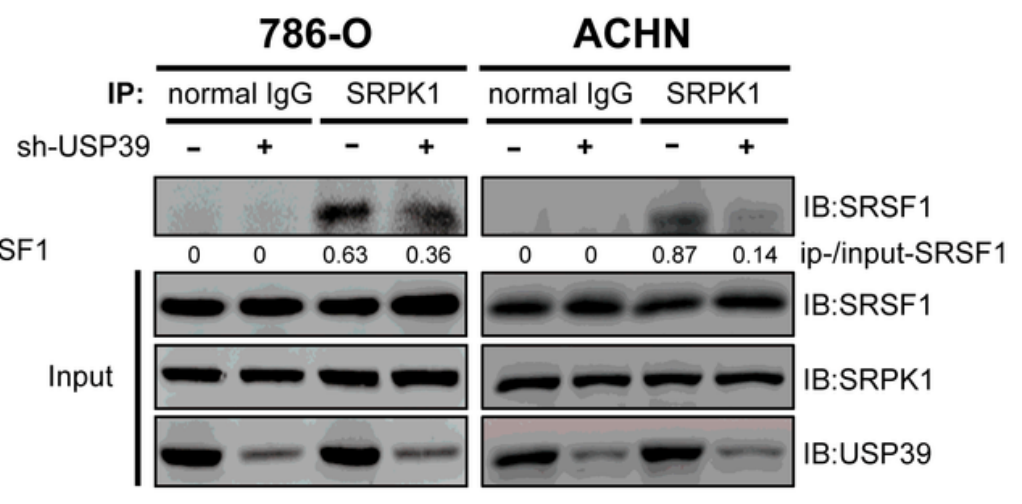

:USP39

\section{$\mathbf{E}$}

D

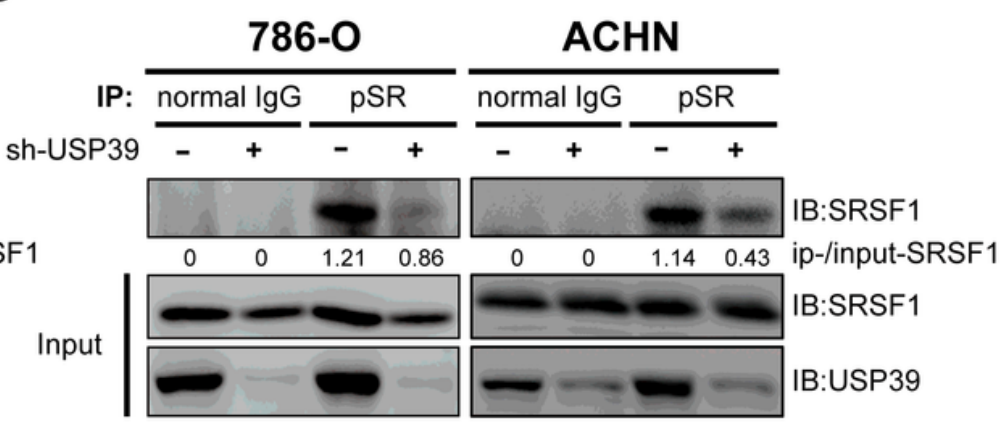

IB:SRSF1 ip-/input-SRSF1 IB:SRSF1 IB:USP39

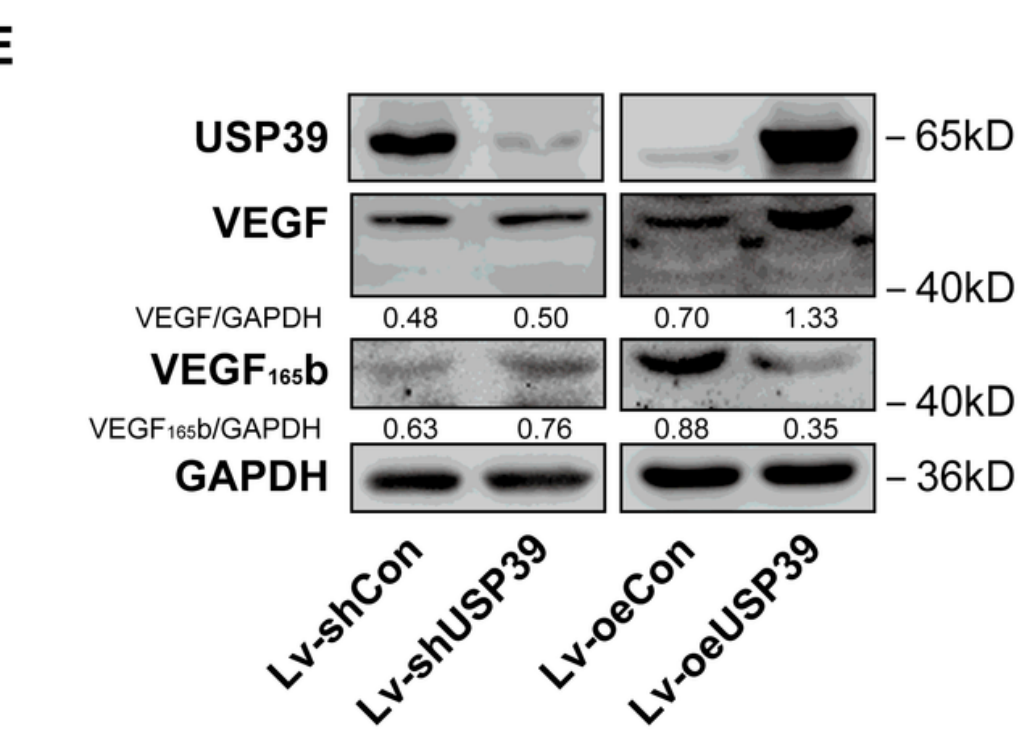

Figure 6

The expression level of USP39 affects the interaction between SRPK1 and SRSF1 and the effect of USP39 expression on VEGF-A variable splicing. (A) USP39 overexpression promoted the interaction between SRPK1 and SRSF1. (B) USP39 knockdown inhibited the interaction between SRPK1 and SRSF1. (C) USP39 overexpression promoted SRPK1 and SRPK1, and phosphorylation of SRSF1. (D) USP39 
knockdown inhibited SRPK1 phosphorylation of SRSF1. (E) Total VEGF and VEGF-165b expression after USP39 knockdown or overexpression.

\section{Supplementary Files}

This is a list of supplementary files associated with this preprint. Click to download.

- supplementary.xlsx

- OnlineGraphicalabstract.Png 